\title{
A greedoid and a matroid inspired by Bhargava's $p$-orderings
}

\author{
Darij Grinberg* \\ Mathematics Department \\ Drexel University \\ Philadelphia, PA 19104, U.S.A. \\ darijgrinberg@gmail.com / http://www.cip.ifi.lmu.de/ grinberg/ \\ Fedor Petrov \\ St. Petersburg State University and \\ St. Petersburg Department \\ Steklov Mathematical Institute of Russian Academy of Sciences \\ St. Petersburg, Russia. \\ fedyapetrov@gmail.com / http://math-cs.spbu.ru/people/petrov-f-v/ \\ Submitted: Oct 7, 2019; Accepted: Jun 10, 2021; Published: Jul 2, 2021 \\ (C) The authors. Released under the CC BY license (International 4.0).
}

\begin{abstract}
Consider a finite set $E$. Assume that each $e \in E$ has a "weight" $w(e) \in \mathbb{R}$ assigned to it, and any two distinct $e, f \in E$ have a "distance" $d(e, f)=d(f, e) \in \mathbb{R}$ assigned to them, such that the distances satisfy the ultrametric triangle inequality $d(a, b) \leqslant \max \{d(a, c), d(b, c)\}$. We look for a subset of $E$ of given size with maximum perimeter (where the perimeter is defined by summing the weights of all elements and their pairwise distances). We show that any such subset can be found by a greedy algorithm (which starts with the empty set, and then adds new elements one by one, maximizing the perimeter at each step). We use this to define numerical invariants, and also to show that the maximum-perimeter subsets of all sizes are the feasible sets of a strong greedoid, and the maximum-perimeter subsets of any given size are the bases of a matroid. This essentially generalizes the " $P$-orderings" constructed by Bhargava in order to define his generalized factorials, and is also similar to the strong greedoid of maximum diversity subsets in phylogenetic trees studied by Moulton, Semple and Steel.

We further discuss some numerical invariants of $E, w, d$ stemming from this construction, as well as an analogue where maximum-perimeter subsets are replaced by maximum-perimeter tuples (i.e., elements can appear multiple times).
\end{abstract}

Mathematics Subject Classifications: 05B35, 90C27, 92B10

${ }^{*}$ DG thanks the Mathematisches Forschungsinstitut Oberwolfach for its hospitality. 


\section{Contents}

1 Introduction 2

2 The setup 3

2.1 Defining ultra triples . . . . . . . . . . . . . . . . . 3

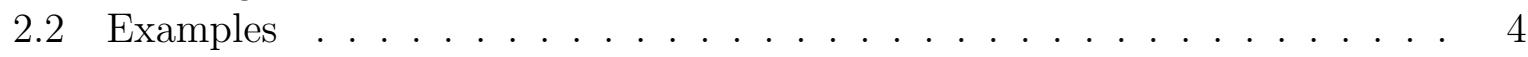

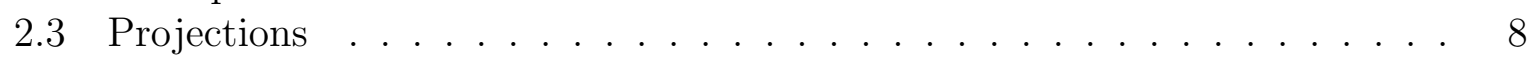

3 Perimeters and greedy $m$-permutations 9

3.1 The perimeter of an $m$-set . . . . . . . . . . . . . . . . . . . . 9

3.2 Defining greedy $m$-permutations . . . . . . . . . . . . . . . . . . 9

3.3 Examples of greediness . . . . . . . . . . . . . . . . . . . . . . . . . . . . . . . . . .

3.4 Basic properties of greediness . . . . . . . . . . . . . . 10

4 The main theorems $\quad 12$

5 The $\nu_{k}^{\circ}(C)$ invariants $\quad 15$

6 The greedoid 16

6.1 Defining greedoids and strong greedoids . . . . . . . . . . . . . 16

6.2 The Bhargava greedoid . . . . . . . . . . . . . . . 17

7 The matroid $\quad 20$

7.1 Defining matroids . . . . . . . . . . . . . . . . 20

7.2 Matroids from strong greedoids . . . . . . . . . . . . . . 21

8 Greedy subsequences 23

8.1 Full ultra triples . . . . . . . . . . . . . . . . . . . 23

8.2 Further definitions . . . . . . . . . . . . . . . . . . 25

8.3 Main analogues . . . . . . . . . . . . . . . . . . . . 26

8.4 The clone construction . . . . . . . . . . . . . . . 27

8.5 Proofs of the analogues . . . . . . . . . . . . . . . . . 28

9 Relation to Bhargava's $P$-orderings $\quad 31$

10 Appendix: Greediness of $(1,2, \ldots, m)$ for $p$-adic metrics 34

\section{Introduction}

In this paper, we study a combinatorial setting consisting of a finite set $E$ with a "weight function" $w: E \rightarrow \mathbb{R}$ and a (symmetric) "distance function" $d: E \underline{\times} E \rightarrow \mathbb{R}$ (where $E \underline{\times} E=\{(e, f) \in E \times E \mid e \neq f\})$ satisfying the ultrametric triangle inequality. This generalizes the notion of an ultrametric space. Given any finite subset $A$ of $E$, we can define the perimeter of $A$ to be the sum of the weights and of the pairwise distances of the 
elements of $A$. Given an integer $k \geqslant 0$ and a finite subset $C$ of $E$, we show (Theorem 21) that if we want to construct a $k$-element subset of $C$ having maximum perimeter, we can do so by a greedy algorithm (i.e., by starting with the empty set and repeatedly adding new elements that increase the perimeter as much as possible), and that every maximumperimeter $k$-element subset of $C$ can be constructed through this algorithm (Theorem 22). We furthermore show that these maximum-perimeter $k$-element subsets are the bases of a matroid (when $k$ is fixed) and the feasible sets of a strong greedoid (when $k$ ranges over all nonnegative integers). In a followup paper [8], this strong greedoid is studied from an algebraic viewpoint, which also addresses questions of its (linear) representability.

Our greedy construction of maximum-perimeter subsets is inspired by Manjul Bhargava's concept of a $P$-ordering ([2, Section 2]), which laid the foundation for his theory of generalized factorials (see [3, Section 4] and [4, Section 2]); we connect the two notions (in Section 9) and obtain new proofs of two results from [2, Section 2].

A similar problem - also leading to a strong greedoid - has appeared in the mathematical biology literature: Given a phylogenetic tree $T$ and an integer $k$, the problem asks to find a set of $k$ leaves of $T$ having maximum "phylogenetic diversity" (i.e., the total weight of the edges of the subtree that connects these $k$ leaves). In [11], Moulton, Semple and Steel show that such diversity-maximizing $k$-element sets form a strong greedoid, just as our maximum-perimeter subsets do. The similarity does not end here: Phylogenetic trees are close relatives of ultra triples (and can be translated to and from the latter without much loss of information). However, the strong greedoid of Moulton, Semple and Steel is not the same as ours, since perimeter (when restated in terms of the phylogenetic tree) is not the same as phylogenetic diversity ${ }^{1}$. It is an interesting question to what extent these two problems can be reconciled, and perhaps a more general class of optimization problems on phylogenetic trees (or ultra triples) can be shown to lead to a strong greedoid.

\section{The setup}

\subsection{Defining ultra triples}

Let $E$ be a set. We shall use $E$ as our ground set throughout this paper.

We shall refer to the elements of $E$ as points.

For a nonnegative integer $m$, an $m$-set means a subset $A$ of $E$ which consists of $|A|=m$ elements. If $B \subseteq E$ is any subset and $m$ is a nonnegative integer, then an $m$-subset of $B$ means an $m$-element subset of $B$.

Define the set $E \underline{\times} E$ by

$$
E \underline{\times} E=\{(e, f) \in E \times E \mid e \neq f\} .
$$

Thus, $E \times E$ is the set of all ordered pairs $(e, f)$ of two distinct elements of $E$.

\footnotetext{
${ }^{1}$ Roughly speaking, in a star-shaped phylogenetic tree with 1 internal vertex and $p$ leaves, the perimeter of a $k$-leaf set is quadratic in $k$, while its phylogenetic diversity is linear in $k$. Also, our Lemma 28, while being an analogue of [11, Lemma 3.1], differs from the latter in that it requires $|B|=|A|+1$ rather than $|B|>|A|$ (and indeed, the latter requirement would not suffice).
} 
Assume that we are given a function $w: E \rightarrow \mathbb{R}$. In other words, each point $a \in E$ has a real-valued weight $w(a)$ assigned to it.

Assume further that we are given a function $d: E \underline{x} E \rightarrow \mathbb{R}$, which we will call the distance function. Thus, any two distinct points $a, b \in E$ have a real-valued distance $d(a, b)$. We assume that this distance function has the following properties:

- It is symmetric: that is, $d(a, b)=d(b, a)$ for any two distinct $a, b \in E$.

- It satisfies the following inequality:

$$
d(a, b) \leqslant \max \{d(a, c), d(b, c)\}
$$

for any three distinct $a, b, c \in E$.

(The inequality (1) is commonly known as the ultrametric triangle inequality ${ }^{2}$; but unlike the distance function of an ultrametric space, our $d$ can take negative values. The values of $w$ are completely unrestrained.)

Such a structure $(E, w, d)$ will be called an ultra triple.

From now on, we shall always be considering an ultra triple $(E, w, d)$ (unless stated otherwise).

\subsection{Examples}

We shall now provide a few examples of ultra triples. In each case, the proof that our triple is indeed an ultra triple is easy and left to the reader.

Example 1. For this example, we let $E$ be an arbitrary set, and we define the distances $d(a, b)$ as follows:

$$
d(a, b)=1 \quad \text { for all }(a, b) \in E \underline{x} E .
$$

We define the weights $w(a)$ arbitrarily. Then, $(E, w, d)$ is an ultra triple.

Example 2. For this example, we let $E=\{1,2,3,4,5\}$, and we define the distances $d(a, b)$ as follows:

$$
d(a, b)=\left\{\begin{array}{lll}
1, & \text { if } a \equiv b & \bmod 2 ; \\
2, & \text { if } a \neq \equiv b & \bmod 2
\end{array} \quad \text { for all }(a, b) \in E \underline{\times} E .\right.
$$

We define the weights $w(a)$ arbitrarily. Then, $(E, w, d)$ is an ultra triple.

Example 3. For this example, we fix two reals $\varepsilon$ and $\alpha$ with $\varepsilon \leqslant \alpha$. Furthermore, we fix an integer $m$ and a subset $E$ of $\mathbb{Z}$. We define the distance function $d: E \underline{\times} E \rightarrow \mathbb{R}$ by setting

$$
d(a, b)=\left\{\begin{array}{lll}
\varepsilon, & \text { if } a \equiv b & \bmod m ; \\
\alpha, & \text { if } a \neq \equiv b & \bmod m
\end{array} \quad \text { for all }(a, b) \in E \times E .\right.
$$

We define the weights $w(a)$ arbitrarily. Then, $(E, w, d)$ is an ultra triple.

\footnotetext{
${ }^{2}$ It can be restated as "the longest two sides of a triangle are always equal in length". (Here, a triangle means a 3 -subset of $E$; its sides are its 2-subsets; the length of a side $\{u, v\}$ is $d(u, v)$.)
} 
Note that Example 2 is the particular case of Example 3 obtained by setting $\varepsilon=1$, $\alpha=2, m=2$ and $E=\{1,2,3,4,5\}$.

Example 4. For this example, we fix a prime number $p$ and a subset $E$ of $\mathbb{Z}$, and we define the distances $d(a, b)$ as follows:

$$
d(a, b)=p^{-v_{p}(a-b)} \quad \text { for all }(a, b) \in E \times E .
$$

Here, for any nonzero integer $m$, we let $v_{p}(m)$ denote the $p$-adic valuation of $m$ (that is, the largest nonnegative integer $k$ such that $\left.p^{k} \mid m\right)$. The distance function $d: E \times E \rightarrow \mathbb{R}$ is called the $p$-adic metric. We define the weights $w(a)$ arbitrarily. Then, $(E, w, d)$ is an ultra triple.

Example 5. For this example, we fix a prime number $p$ and a subset $E$ of $\mathbb{Z}$.

For any nonzero integer $m$, we define $v_{p}(m)$ as in Example 4 . We define a map $d^{\prime}: E \underline{\times} E \rightarrow \mathbb{R}$ by setting

$$
d^{\prime}(a, b)=-v_{p}(a-b) \quad \text { for all }(a, b) \in E \underline{\times} E .
$$

We define the weights $w(a)$ arbitrarily. Then, $\left(E, w, d^{\prime}\right)$ is an ultra triple.

Most of the examples above are particular cases of a more general construction:

Example 6. Let $\mathbb{N}=\{0,1,2, \ldots\}$. Let $c: \mathbb{N} \rightarrow \mathbb{R}$ be a weakly decreasing function.

Fix a sequence $\mathbf{r}=\left(r_{0}, r_{1}, r_{2}, \ldots\right)$ of integers such that $r_{0}\left|r_{1}\right| r_{2} \mid \cdots$.

For each $x \in \mathbb{Z}$, define an element $v_{\mathbf{r}}(x) \in \mathbb{N}$ by

$$
v_{\mathbf{r}}(x)=\max \left\{i \in \mathbb{N} \text { such that } r_{i} \mid x\right\},
$$

assuming that this maximum exists. (Otherwise, leave $v_{\mathbf{r}}(x)$ undefined.)

Let $E$ be a subset of $\mathbb{Z}$. Define a distance function $d: E \underline{\times} E \rightarrow \mathbb{R}$ by setting

$$
d(a, b)=c\left(v_{\mathbf{r}}(a-b)\right) \quad \text { for all }(a, b) \in E \underline{\times} E .
$$

Assume that this is well-defined (i.e., the values $v_{\mathbf{r}}(a-b)$ are well-defined for all $(a, b) \in$ $E \underline{\times} E)$. We define the weights $w(a)$ arbitrarily. Then, $(E, w, d)$ is an ultra triple.

Example 3 is obtained from Example 6 by setting $r_{0}=1$ and $r_{1}=m$ and $r_{2}=r_{3}=$ $r_{4}=\cdots=0$ and $c(0)=\alpha$ and $c(1)=\varepsilon$ (defining the remaining values of $c$ arbitrarily to be weakly decreasing). Example 4 is obtained from Example 6 by setting $r_{i}=p^{i}$ and $c(n)=p^{-n}$ (indeed, if we set $r_{i}=p^{i}$, then $v_{\mathbf{r}}(m)=v_{p}(m)$ for each nonzero $m \in \mathbb{Z}$ ). Likewise, Example 5 is obtained from Example 6 by setting $r_{i}=p^{i}$ and $c(n)=-n$.

An even more general (and simpler) example of an ultra triple (more precisely, of a distance function satisfying (1)) can be obtained from a hierarchy of equivalence relations: 
Example 7. Let $E$ be a set. Let $\underset{0}{\sim} \underset{1}{\sim} \underset{2}{\sim}, \ldots$ be equivalence relations on $E$. Assume that: ${ }^{3}$

(A) Every $e, f \in E$ satisfy $e \underset{0}{\sim} f$.

(B) If some $e, f \in E$ and $i>0$ satisfy $e \underset{i}{\sim} f$, then $e \underset{i-1}{\sim} f$.

(C) If $e, f \in E$ are distinct, then there exists some $i \geqslant 0$ such that we don't have $e \underset{i}{\sim} f$.

Let $c: \mathbb{N} \rightarrow \mathbb{R}$ be a weakly decreasing function.

Define a distance function $d: E \underline{\times} E \rightarrow \mathbb{R}$ by

$$
d(e, f)=c(\max \{i \geqslant 0 \mid e \underset{i}{\sim} f\}) \quad \text { for all }(e, f) \in E \underline{\times} E .
$$

We define the weights $w(a)$ arbitrarily. Then, $(E, w, d)$ is an ultra triple.

We can obtain Example 6 from Example 7 by defining the relation $\underset{i}{\sim}$ to be congruence modulo $r_{i}$. (The assumption that the $v_{\mathbf{r}}(a-b)$ are well-defined in Example 6 ensures that assumptions (A), (B) and (C) of Example 7 are satisfied.)

Hierarchical taxonomies can be viewed as sets $E$ equipped with sequences $\underset{0}{\sim}, \underset{1}{\sim}, \underset{2}{\sim}, \ldots$ of equivalence relations (usually finite, however) satisfying assumptions (A), (B) and (C) of Example 7. For example:

Example 8. Let $E$ be the set of all living organisms. Define equivalence relations $\underset{0}{\sim}, \sim_{1}$ $\underset{2}{\sim}, \ldots$ on $E$ as follows:

$$
\begin{aligned}
& (e \underset{0}{\sim} f) \text { always holds; } \\
& (e \underset{1}{\sim} f) \Longleftrightarrow(e \text { and } f \text { belong to the same domain }) ; \\
& (e \underset{2}{\sim} f) \Longleftrightarrow(e \text { and } f \text { belong to the same kingdom }) ; \\
& \quad \vdots \\
& (e \underset{7}{\sim} f) \Longleftrightarrow(e \text { and } f \text { belong to the same genus }) ; \\
& (e \underset{8}{\sim} f) \Longleftrightarrow(e \text { and } f \text { belong to the same species }) ;
\end{aligned}
$$

\footnotetext{
${ }^{3}$ These three assumptions can be restated in terms of the partition lattice on $E$, which is the lattice of all set partitions of $E$ (see, e.g., [13, Section 1.7]). Indeed, it is well-known that the equivalence relations on $E$ are in bijection with the set partitions of $E$. Our sequence $\underset{0}{\sim}, \underset{1}{\sim}, \underset{2}{\sim}, \ldots$ of equivalence relations thus corresponds to a sequence $P_{0}, P_{1}, P_{2}, \ldots$ of set partitions of $E$. The three assumptions below thus say that $P_{0}$ is the trivial partition; that we have $P_{0} \geqslant P_{1} \geqslant P_{2} \geqslant \cdots$ (meaning that each partition $P_{i}$ refines $\left.P_{i-1}\right)$; and that the meet $\bigwedge_{i=0}^{\infty} P_{i}$ is the partition of $E$ into singletons.
} 


$$
(e \underset{i}{\sim} f) \Longleftrightarrow(e=f) \quad \text { for all } i \geqslant 9
$$

(following the taxonomic ranks of biology). Then, assumptions (A), (B) and (C) of Example 7 are satisfied.

Example 8 yields not so much a genuine biological application of our theory as it does a helpful mental model for it. A less naive (if still simplified) model of the interrelation of organisms is provided by phylogenetic trees [11] - rooted trees (in the combinatorial sense) whose vertices correspond to organisms or species, and whose edges signify relationships of ancestry. Often the edges are equipped with weights (or, better, lengths) encoding the evolutionary distance between parent and child vertices. This model, too, leads to an ultra triple. At the mathematical heart of this construction is the following example:

Example 9. A tree is a connected finite undirected graph that has no cycles. (Thus, our trees are unrooted and have no order-like structures assigned to them.)

Let $T$ be a tree. For each edge $e$ of $T$, let $\lambda(e)$ be an nonnegative real. We shall call this real the weight of $e$.

For any vertices $u$ and $v$ of $T$, let $\lambda(u, v)$ denote the sum of the weights of all edges on the (unique) path from $u$ to $v$. Note that this $\lambda(u, v)$ generalizes the usual (graphtheoretical) distance between $u$ and $v$; indeed, if $\lambda(e)=1$ for each edge $e$ of $T$, then $\lambda(u, v)$ is the distance between $u$ and $v$ (that is, the length of the unique path from $u$ to $v)$.

A known result (the "four-point condition") says that if $x, y, z, w$ are four vertices of $T$, then the two largest of the three numbers

$$
\lambda(x, y)+\lambda(z, w), \quad \lambda(x, z)+\lambda(y, w), \quad \text { and } \lambda(x, w)+\lambda(y, z)
$$

are equal. In the particular case when each edge of $T$ has weight 1 , this is a standard exercise in graph theory (see, e.g., https://math. stackexchange.com/questions/2899278); most of its solutions generalize to the case of arbitrary weights. We can use this to define an ultra triple as follows:

Fix any vertex $r$ of $T$. Let $E$ be any subset of the vertex set of $T$. We define a map $w: E \rightarrow \mathbb{R}$ by setting

$$
w(x)=\lambda(x, r) \quad \text { for each } x \in E .
$$

We define a map $d: E \underline{\times} E \rightarrow \mathbb{R}$ by setting

$$
d(x, y)=\lambda(x, y)-\lambda(x, r)-\lambda(y, r) \quad \text { for each }(x, y) \in E \underline{\times} E .
$$

Then, $(E, w, d)$ is an ultra triple.

Note that our special choice of $w$ was not necessary for this (any function $w: E \rightarrow \mathbb{R}$ would have worked), but it has the advantage that $\lambda(x, y)=w(x)+w(y)+d(x, y)$ for any distinct $x, y \in E$. The right hand side of this equality will later be called the perimeter $\operatorname{PER}\{x, y\}$ of the set $\{x, y\}$. 


\subsection{Projections}

Let us now return to the setting of an arbitrary ultra triple $(E, w, d)$.

Definition 10. Let $C \subseteq E$ be a non-empty subset. Let $v \in E$ be any point.

We define a subset $\operatorname{proj}_{C}(v)$ of $C$ as follows:

- If $v \in C$, then we define $\operatorname{proj}_{C}(v)$ to be the one-element set $\{v\}$.

- If $v \notin C$, then we define $\operatorname{proj}_{C}(v)$ to be the set of all $c \in C$ that minimize the distance $d(v, c) \quad 4$.

The elements of $\operatorname{proj}_{C}(v)$ will be called the projections of $v$ onto $C$.

The following is easy to see and will be used without explicit mention:

Proposition 11. Let $C \subseteq E$ be a finite non-empty subset. Let $v \in E$ be any point. Then, there exists at least one projection of $v$ onto $C$.

Example 12. Let $(E, w, d)$ be as in Example 2. Then, the projections of 2 onto $\{1,3\}$ are 1 and 3, while the only projection of 2 onto $\{1,3,4\}$ is 4 .

In Example 9, a projection of a $v \notin C$ onto a subset $C$ is usually called a "closest relative of $v$ in $C^{\prime \prime}$.

The crucial property of projections if the following:

Lemma 13. Assume that $C \subseteq E$ is a non-empty subset and $v \in E$ is any point. Let $u$ be a projection of $v$ onto $C$.

(a) If $v \in C$, then $u=v$.

(b) If $x \in C$ satisfies $x \neq u$, then $x \neq v$.

(c) Let $x \in C$ be such that $x \neq u$. Then, $d(u, x) \leqslant d(v, x)$.

Proof. We have $u \in \operatorname{proj}_{C}(v)$ (since $u$ is a projection of $v$ onto $C$ ).

(a) follows from the definition. So does (b): if we had $x=v$, then we would have $v=x \in C$ and therefore $u=v$ (by (a)).

(c) By (b), we have $x \neq v$. Hence, $d(v, x)$ is well-defined. Also, $d(u, x)$ is well-defined (since $x \neq u$ ).

If $u=v$, then $d(u, x)=d(v, x)$. Thus we WLOG assume that $u \neq v$. Hence, part (a) entails $v \notin C$. The points $v, u, x$ are distinct, and so by (1) we have

$$
d(u, x) \leqslant \max (d(v, u), d(v, x))=d(v, x) .
$$

(The last equality is equivalent to $d(v, u) \leqslant d(v, x)$, which follows from the definition of projection.)

\footnotetext{
${ }^{4}$ This distance $d(v, c)$ is well-defined, since $v \neq c$ (because $v \notin C$ and $c \in C$ ).
} 


\section{Perimeters and greedy $m$-permutations}

\subsection{The perimeter of an $m$-set}

For any finite subset $A \subseteq E$, we define its perimeter $\operatorname{PER}(A)$ by

$$
\operatorname{PER}(A):=\sum_{a \in A} w(a)+\sum_{\substack{\{a, b\} \subseteq A ; \\ a \neq b}} d(a, b) .
$$

The second sum here is taken over all unordered pairs $a \neq b$ of distinct elements of $A$. (This is well-defined, since $d(a, b)=d(b, a)$ for any distinct $a, b \in E$.)

Example 14. Let $(E, w, d)$ be as in Example 2. Then,

$$
\operatorname{PER}\{1,2,3\}=w(1)+w(2)+w(3)+\underbrace{d(1,2)}_{=2}+\underbrace{d(1,3)}_{=1}+\underbrace{d(2,3)}_{=2} .
$$

\subsection{Defining greedy $m$-permutations}

Definition 15. Let $C \subseteq E$ be any subset, and let $m$ be a nonnegative integer.

A greedy m-permutation of $C$ is a list $\left(c_{1}, c_{2}, \ldots, c_{m}\right)$ of $m$ distinct elements of $C$ such that for each $i \in\{1,2, \ldots, m\}$ and each $x \in C \backslash\left\{c_{1}, c_{2}, \ldots, c_{i-1}\right\}$, we have

$$
\operatorname{PER}\left\{c_{1}, c_{2}, \ldots, c_{i}\right\} \geqslant \operatorname{PER}\left\{c_{1}, c_{2}, \ldots, c_{i-1}, x\right\} .
$$

Thus, roughly speaking, a greedy $m$-permutation is an ordered sample of $m$ distinct elements of $C$ such that at each step of the sampling procedure, the new element is chosen in such a way as to maximize the perimeter of the sample. This procedure can be viewed as a greedy algorithm to construct an $m$-subset of $C$ that has maximum perimeter. As we shall see in Theorem 21, this algorithm indeed succeeds at constructing such a subset.

\subsection{Examples of greediness}

Example 16. Let $(E, w, d)$ be as in Example 2. Assume that $w(a)=0$ for all $a \in E$.

Then, $(1,2),(2,1)$ and $(5,4)$ (and several others) are greedy 2-permutations of $E$. Actually, a pair $(i, j)$ of elements of $E$ is a greedy 2-permutation of $E$ if and only if $i \neq \equiv j$ $\bmod 2$.

Also, $(1,3)$ is a greedy 2-permutation of $\{1,3,5\}$, but not of $E$ (because we have $\operatorname{PER}\{1,3\}<\operatorname{PER}\{1,2\})$.

Also, $(1,2,3,4,5)$ is a greedy 5 -permutation of $E$, but $(1,2,3,5,4)$ is not (since we have PER $\{1,2,3,5\}<\operatorname{PER}\{1,2,3,4\})$.

Example 17. Let $E$ be the set $\{1,2,3,4,5,6\}$. Fix five reals $\alpha, \lambda, \kappa, \varepsilon, \delta$ such that $\lambda$ and $\kappa$ are both smaller than $\alpha$ and both larger than each of $\varepsilon$ and $\delta$. For any distinct $a, b \in E$, we define the distance $d(a, b)$ by the following rule:

- If $a \not \equiv b \bmod 2$, then $d(a, b)=\alpha$. 
- If $a=1$ and $b \in\{3,5\}$, then $d(a, b)=\lambda$.

- If $a=2$ and $b \in\{4,6\}$, then $d(a, b)=\kappa$.

- If $a=3$ and $b=5$, then $d(a, b)=\varepsilon$.

- If $a=4$ and $b=6$, then $d(a, b)=\delta$.

- Otherwise, $d(a, b)=d(b, a)$.

Set $w(a)=0$ for all $a \in E$.

It is easy to check that $(E, w, d)$ is an ultra triple.

The pair $(1,2)$ is always a greedy 2-permutation.

The 4-tuple $(1,2,3,4)$ is a greedy 4 -permutation if and only if $\lambda \geqslant \kappa$. The 4-tuple $(1,2,4,3)$ is a greedy 4 -permutation if and only if $\kappa \geqslant \lambda$.

The 5-tuple $(1,2,3,4,5)$ is a greedy 5 -permutation if and only if $\lambda \geqslant \kappa$ and $\lambda+\varepsilon \geqslant \kappa+\delta$.

The 5-set $\{1,2,3,4,5\}$ has maximum perimeter among all 5 -sets if and only if $\lambda+\varepsilon \geqslant$ $\kappa+\delta$.

This example illustrates that greedy permutations and maximum-perimeter sets depend not just on the order relations between the distances of the points, but also on the order relations between sums of these distances.

Example 18. For this example, we fix a prime number $p$ and a nonnegative integer $m$. We let $E$ be any subset of $\mathbb{Z}$ that contains $1,2, \ldots, m$. We define $d: E \underline{\times} E \rightarrow \mathbb{R}$ as in Example 4. We define $d^{\prime}: E \times E \rightarrow \mathbb{R}$ as in Example 5. We define $w: E \rightarrow \mathbb{R}$ by setting $w(e)=0$ for all $e \in E$.

Then, $(1,2, \ldots, m)$ is a greedy $m$-permutation of $E$ both for the ultra triple $(E, w, d)$ and for the ultra triple $\left(E, w, d^{\prime}\right)$.

We relegate the proof of this claim to Section 10, as we shall not use it.

Example 19. Example 18 might suggest that the ultra triples $(E, w, d)$ and $\left(E, w, d^{\prime}\right)$ (defined in that example) have the same greedy $m$-permutations in general. This is not the case. For instance, set $p=2$ and $E=\{0,1,2,9,17,128\}$. Define $d, d^{\prime}$ and $w$ as in Example 18.

Now it is easy to check that $(2,9,17,0,1)$ is a greedy 5 -permutation for $\left(E, w, d^{\prime}\right)$ but not for $(E, w, d)$, while $(2,9,17,0,128)$ is a greedy 5 -permutation for $(E, w, d)$ but not for $\left(E, w, d^{\prime}\right)$.

Moreover, the 5-set $\{2,9,17,0,1\}$ has maximum perimeter for $\left(E, w, d^{\prime}\right)$ but not for $(E, w, d)$, while the 5 -set $\{2,9,17,0,128\}$ has maximum perimeter for $(E, w, d)$ but not for $\left(E, w, d^{\prime}\right)$.

\subsection{Basic properties of greediness}

We will use the following shorthand notations: If $S$ is a subset of $E$, and if $e \in E$, then $S \cup e$ and $S \backslash e$ will stand for the subsets $S \cup\{e\}$ and $S \backslash\{e\}$, respectively. Set operations 
like $\cup$ and $\backslash$ shall be read in a left-associative way; thus, e.g., the expression " $S \cup e \backslash f$ " shall be understood as $(S \cup e) \backslash f$.

Let us observe some easy consequences of the definition of greedy permutations (which will be later used without mention):

Proposition 20. Let $C$ be a subset of $E$. Let $m$ be a nonnegative integer.

(a) If a greedy m-permutation of $C$ exists, then $m \leqslant|C|$.

(b) If $\left(c_{1}, c_{2}, \ldots, c_{m}\right)$ is a greedy m-permutation of $C$, then $\left\{c_{1}, c_{2}, \ldots, c_{k}\right\}$ is a $k$-subset of $C$ for each $k \in\{0,1, \ldots, m\}$.

(c) If $\left(c_{1}, c_{2}, \ldots, c_{m}\right)$ is a greedy m-permutation of $C$, then $\left\{c_{1}, c_{2}, \ldots, c_{k-1}\right\}=\left\{c_{1}, c_{2}, \ldots, c_{k}\right\} \backslash c_{k}$ for each $k \in\{1,2, \ldots, m\}$.

(d) If $|C|=m$, then any greedy $m$-permutation of $C$ must be a list of all the $m$ elements of $C$.

(e) If $C$ is finite and satisfies $m \leqslant|C|$, then there exists a greedy m-permutation of $C$.

(f) Assume that $C$ is finite and satisfies $m \leqslant|C|$. Let $n$ be an integer such that $m \geqslant$ $n \geqslant 0$.

If $\left(c_{1}, c_{2}, \ldots, c_{n}\right)$ is a greedy $n$-permutation of $C$, then we can find $m-n$ elements $c_{n+1}, c_{n+2}, \ldots, c_{m}$ of $C$ such that $\left(c_{1}, c_{2}, \ldots, c_{m}\right)$ is a greedy m-permutation of $C$.

(g) Let $n$ be an integer such that $m \geqslant n \geqslant 0$.

If $\left(c_{1}, c_{2}, \ldots, c_{m}\right)$ is a greedy m-permutation of $C$, then $\left(c_{1}, c_{2}, \ldots, c_{n}\right)$ is a greedy $n$-permutation of $C$.

Proof. Parts (a), (b), (c), (d) and (g) are straightforward.

(f) Assume that $\left(c_{1}, c_{2}, \ldots, c_{n}\right)$ is a greedy $n$-permutation of $C$. Then, we construct $c_{n+1}, c_{n+2}, \ldots, c_{m}$ recursively as follows:

- For each $i=n+1, n+2, \ldots, m$, we assume that $c_{1}, c_{2}, \ldots, c_{i-1}$ have already been defined; we then choose an element $c_{i} \in C \backslash\left\{c_{1}, c_{2}, \ldots, c_{i-1}\right\}$ that maximizes the perimeter PER $\left\{c_{1}, c_{2}, \ldots, c_{i}\right\}$. (If there are several such elements, then we choose any of them.)

This procedure can be carried out since $C$ is finite and $m \leqslant|C|$. By construction, it produces a greedy $m$-permutation $\left(c_{1}, c_{2}, \ldots, c_{m}\right)$ of $C$. This proves part (f).

Part (e) is the particular case of part (f) for $n=0$ (since the empty list is a greedy 0-permutation).

The procedure used in the proof of Proposition 20 (f) also works for infinite $C$ as long as the maxima exist. 


\section{The main theorems}

We now state two central theorems for this paper:

Theorem 21. Let $C \subseteq E$ be any subset, and let $m$ be a nonnegative integer.

Let $\left(c_{1}, c_{2}, \ldots, c_{m}\right)$ be any greedy m-permutation of $C$.

Then, for each $k \in\{0,1, \ldots, m\}$, the set $\left\{c_{1}, c_{2}, \ldots, c_{k}\right\}$ has maximum perimeter among all $k$-subsets of $C$.

Theorem 22. Let $C \subseteq E$ be any finite subset, and let $m$ be a nonnegative integer such that $|C| \geqslant m$. Let $k \in\{0,1, \ldots, m\}$.

Let $A$ be a $k$-subset of $C$ having maximum perimeter (among the $k$-subsets of $C$ ). Then, there exists a greedy m-permutation $\left(v_{1}, v_{2}, \ldots, v_{m}\right)$ of $C$ such that $A=\left\{v_{1}, v_{2}, \ldots, v_{k}\right\}$.

Proof of Theorem 21. The points $c_{1}, c_{2}, \ldots, c_{m}$ are distinct (by the definition of a greedy $m$-permutation).

Fix $k \in\{0,1, \ldots, m\}$. Then, $\left\{c_{1}, c_{2}, \ldots, c_{k}\right\}$ is a $k$-subset of $C$ (by Proposition 20 (b)). It remains to prove that every $k$-subset $A$ of $C$ satisfies $\operatorname{PER}(A) \leqslant \operatorname{PER}\left\{c_{1}, c_{2}, \ldots, c_{k}\right\}$.

Let $A$ be any $k$-subset of $C$. We shall show that $\operatorname{PER}(A) \leqslant \operatorname{PER}\left\{c_{1}, c_{2}, \ldots, c_{k}\right\}$. This will clearly prove Theorem 21 .

We define a list $\left(v_{1}, v_{2}, \ldots, v_{k}\right)$ of $k$ elements of $A$ recursively as follows: For each $i=1,2, \ldots, k$, we let $v_{i}$ be a projection of $c_{i}$ onto $A \backslash\left\{v_{1}, v_{2}, \ldots, v_{i-1}\right\}$ (assuming that $v_{1}, v_{2}, \ldots, v_{i-1}$ have already been constructed). ${ }^{5}$ (These projections $v_{i}$ exist because of Proposition $11^{6}$; they may be non-unique, but any choice is fine.)

Thus, we get $k$ elements $v_{1}, v_{2}, \ldots, v_{k}$ of $A$. These $k$ elements are distinct (since each $v_{i}$ has been constructed to belong to $\left.A \backslash\left\{v_{1}, v_{2}, \ldots, v_{i-1}\right\}\right)$. Since $|A|=k$, these $k$ distinct elements must cover the whole set $A$. Hence, $\left(v_{1}, v_{2}, \ldots, v_{k}\right)$ is an enumeration of $A$ (that is, a list of distinct elements of $A$ such that $\left.A=\left\{v_{1}, v_{2}, \ldots, v_{k}\right\}\right)$.

Let $j \in\{1,2, \ldots, k\}$. Then,

$$
v_{j} \notin\left\{c_{1}, c_{2}, \ldots, c_{j-1}\right\} .
$$

[Proof of (3): Assume the contrary. Thus, $v_{j} \in\left\{c_{1}, c_{2}, \ldots, c_{j-1}\right\}$, so that $v_{j}=c_{i}$ for some $i<j$. Consider this $i$. Hence, $c_{i}=v_{j} \in\left\{v_{i}, v_{i+1}, \ldots, v_{k}\right\}=A \backslash\left\{v_{1}, v_{2}, \ldots, v_{i-1}\right\}$ (since $\left(v_{1}, v_{2}, \ldots, v_{k}\right)$ is an enumeration of $A$ ). But our recursive definition of $v_{i}$ shows that $v_{i}$ is a projection of $c_{i}$ onto the set $A \backslash\left\{v_{1}, v_{2}, \ldots, v_{i-1}\right\}$. Hence, Lemma 13 (a) (applied to $A \backslash\left\{v_{1}, v_{2}, \ldots, v_{i-1}\right\}, c_{i}$ and $v_{i}$ instead of $C, v$ and $u$ ) yields $v_{i}=c_{i}$ (since $c_{i} \in A \backslash\left\{v_{1}, v_{2}, \ldots, v_{i-1}\right\}$ ). Hence, $v_{i}=c_{i}=v_{j}$, whence $i=j$ (since $v_{1}, v_{2}, \ldots, v_{k}$ are distinct). But this contradicts $i<j$. This contradiction shows that our assumption was false, and thus (3) is proven.]

\footnotetext{
${ }^{5}$ Thus, in particular, $v_{1}$ is a projection of $c_{1}$ onto $A$.

${ }^{6}$ In more detail: Let $i \in\{1,2, \ldots, k\}$, and assume that $v_{1}, v_{2}, \ldots, v_{i-1}$ have already been constructed; we must prove that $v_{i}$ exists. We have $\left|\left\{v_{1}, v_{2}, \ldots, v_{i-1}\right\}\right| \leqslant i-1<i \leqslant k=|A|$; thus, the set $A \backslash$ $\left\{v_{1}, v_{2}, \ldots, v_{i-1}\right\}$ is nonempty. Since this set is furthermore finite, we thus conclude (by Proposition 11) that there exists a projection of $c_{i}$ onto $A \backslash\left\{v_{1}, v_{2}, \ldots, v_{i-1}\right\}$. In other words, $v_{i}$ exists.
} 
Hence, $v_{j} \in A \backslash\left\{c_{1}, c_{2}, \ldots, c_{j-1}\right\} \subseteq C \backslash\left\{c_{1}, c_{2}, \ldots, c_{j-1}\right\}$ (since $A \subseteq C$ ) and therefore PER $\left\{c_{1}, c_{2}, \ldots, c_{j-1}, v_{j}\right\} \leqslant \operatorname{PER}\left\{c_{1}, c_{2}, \ldots, c_{j}\right\}$ by the definition of a greedy $m$ permutation (specifically, by (2)).

But $c_{1}, c_{2}, \ldots, c_{j-1}, v_{j}$ are distinct (since $c_{1}, c_{2}, \ldots, c_{m}$ are distinct, and since $v_{j} \notin$ $\left.\left\{c_{1}, c_{2}, \ldots, c_{j-1}\right\}\right)$, and thus

$$
\begin{aligned}
& \operatorname{PER}\left\{c_{1}, c_{2}, \ldots, c_{j-1}\right\}+w\left(v_{j}\right)+\sum_{i=1}^{j-1} d\left(c_{i}, v_{j}\right)=\operatorname{PER}\left\{c_{1}, c_{2}, \ldots, c_{j-1}, v_{j}\right\} \\
& \leqslant \operatorname{PER}\left\{c_{1}, c_{2}, \ldots, c_{j}\right\}=\operatorname{PER}\left\{c_{1}, c_{2}, \ldots, c_{j-1}\right\}+w\left(c_{j}\right)+\sum_{i=1}^{j-1} d\left(c_{i}, c_{j}\right)
\end{aligned}
$$

(since $c_{1}, c_{2}, \ldots, c_{j}$ are distinct). After cancelling equal terms, this rewrites as

$$
w\left(v_{j}\right)+\sum_{i=1}^{j-1} d\left(c_{i}, v_{j}\right) \leqslant w\left(c_{j}\right)+\sum_{i=1}^{j-1} d\left(c_{i}, c_{j}\right) .
$$

Furthermore, for each $i \in\{1,2, \ldots, j-1\}$, we have $j>i$ and thus

$$
v_{j} \in\left\{v_{i}, v_{i+1}, \ldots, v_{k}\right\}=A \backslash\left\{v_{1}, v_{2}, \ldots, v_{i-1}\right\}
$$

(since $\left(v_{1}, v_{2}, \ldots, v_{k}\right)$ is an enumeration of $A$ ) and $v_{j} \neq v_{i}$ (since $v_{1}, v_{2}, \ldots, v_{k}$ are distinct). Hence, Lemma 13 (c) (applied to $A \backslash\left\{v_{1}, v_{2}, \ldots, v_{i-1}\right\}, c_{i}, v_{i}$ and $v_{j}$ instead of $C, v, u$ and $x$ ) yields

$$
d\left(v_{i}, v_{j}\right) \leqslant d\left(c_{i}, v_{j}\right) \text { for each } i \in\{1,2, \ldots, j-1\}
$$

(since $v_{i}$ is a projection of $c_{i}$ onto the set $A \backslash\left\{v_{1}, v_{2}, \ldots, v_{i-1}\right\}$, whereas $v_{j} \in A \backslash$ $\left\{v_{1}, v_{2}, \ldots, v_{i-1}\right\}$ and $\left.v_{j} \neq v_{i}\right)$.

Now, forget that we fixed $j$. We thus have proven (4) and (5) for each $j \in\{1,2, \ldots, k\}$.

But $\left(v_{1}, v_{2}, \ldots, v_{k}\right)$ is an enumeration of $A$; thus,

$$
\begin{aligned}
\operatorname{PER}(A) & =\sum_{j=1}^{k} w\left(v_{j}\right)+\sum_{1 \leqslant i<j \leqslant k} d\left(v_{i}, v_{j}\right)=\sum_{j=1}^{k}\left(w\left(v_{j}\right)+\sum_{i=1}^{j-1} d\left(v_{i}, v_{j}\right)\right) \\
& \leqslant \sum_{j=1}^{k}\left(w\left(v_{j}\right)+\sum_{i=1}^{j-1} d\left(c_{i}, v_{j}\right)\right) \quad(\text { by }(5)) \\
& \leqslant \sum_{j=1}^{k}\left(w\left(c_{j}\right)+\sum_{i=1}^{j-1} d\left(c_{i}, c_{j}\right)\right) \quad(\text { by }(4)) \\
& =\sum_{j=1}^{k} w\left(c_{j}\right)+\sum_{1 \leqslant i<j \leqslant k} d\left(c_{i}, c_{j}\right)=\operatorname{PER}\left\{c_{1}, c_{2}, \ldots, c_{k}\right\}
\end{aligned}
$$

(since $c_{1}, c_{2}, \ldots, c_{k}$ are distinct). This proves Theorem 21 . 
Proof of Theorem 22. Proposition 20 (e) shows that there exists a greedy $m$-permutation of $C$ (since $C$ is finite and since $|C| \geqslant m$ ). Choose such a greedy $m$-permutation $\left(c_{1}, c_{2}, \ldots, c_{m}\right)$ of $C$. Then, Theorem 21 shows that the set $\left\{c_{1}, c_{2}, \ldots, c_{k}\right\}$ has maximum perimeter among all $k$-subsets of $C$. Hence, $\operatorname{PER}(A)=\operatorname{PER}\left\{c_{1}, c_{2}, \ldots, c_{k}\right\}$ (since the set $A$ also has maximum perimeter among them).

Construct an enumeration $\left(v_{1}, v_{2}, \ldots, v_{k}\right)$ of $A$ as in the proof of Theorem 21 above. In our above proof of Theorem 21, we have proven the inequalities (4) and (5) for all $j \in\{1,2, \ldots, k\}$. But by adding together all these inequalities, we have obtained the inequality $\operatorname{PER}(A) \leqslant \operatorname{PER}\left\{c_{1}, c_{2}, \ldots, c_{k}\right\}$, which must be an equality (since $\operatorname{PER}(A)=$ $\left.\operatorname{PER}\left\{c_{1}, c_{2}, \ldots, c_{k}\right\}\right)$. Thus, all the inequalities (4) and (5) must become equalities (because if adding together a bunch of inequalities produces an equality, then all the inequalities must themselves be equalities).

Hence, for all $j \in\{1,2, \ldots, k\}$, we have

$$
d\left(v_{i}, v_{j}\right)=d\left(c_{i}, v_{j}\right) \quad \text { for each } i \in\{1,2, \ldots, j-1\}
$$

(since (5) become equalities), and thus

$$
\begin{aligned}
w\left(v_{j}\right)+\sum_{i=1}^{j-1} d\left(v_{i}, v_{j}\right) & =w\left(v_{j}\right)+\sum_{i=1}^{j-1} d\left(c_{i}, v_{j}\right) \\
& =w\left(c_{j}\right)+\sum_{i=1}^{j-1} d\left(c_{i}, c_{j}\right)
\end{aligned}
$$

(since (4) become equalities).

Now, fix $p \in\{1,2, \ldots, k\}$. Hence, $p \leqslant k \leqslant m$. The points $c_{1}, c_{2}, \ldots, c_{m}$ are distinct (since $\left(c_{1}, c_{2}, \ldots, c_{m}\right)$ is a greedy $m$-permutation). Thus, the points $c_{1}, c_{2}, \ldots, c_{p}$ are distinct. Also, the points $v_{1}, v_{2}, \ldots, v_{p}$ are distinct (since $v_{1}, v_{2}, \ldots, v_{k}$ are distinct); hence, the definition of a perimeter yields

$$
\begin{aligned}
\operatorname{PER}\left\{v_{1}, v_{2}, \ldots, v_{p}\right\} & =\sum_{j=1}^{p} w\left(v_{j}\right)+\sum_{1 \leqslant i<j \leqslant p} d\left(v_{i}, v_{j}\right) \\
& =\sum_{j=1}^{p}\left(w\left(v_{j}\right)+\sum_{i=1}^{j-1} d\left(v_{i}, v_{j}\right)\right) \\
& =\sum_{j=1}^{p}\left(w\left(c_{j}\right)+\sum_{i=1}^{j-1} d\left(c_{i}, c_{j}\right)\right) \\
& =\sum_{j=1}^{p} w\left(c_{j}\right)+\sum_{1 \leqslant i<j \leqslant p} d\left(c_{i}, c_{j}\right) \\
& =\operatorname{PER}\left\{c_{1}, c_{2}, \ldots, c_{p}\right\}
\end{aligned}
$$

(since the points $c_{1}, c_{2}, \ldots, c_{p}$ are distinct). 
But Theorem 21 (applied to $p$ instead of $k$ ) shows that the set $\left\{c_{1}, c_{2}, \ldots, c_{p}\right\}$ has maximum perimeter among all $p$-subsets of $C$. Hence, the set $\left\{v_{1}, v_{2}, \ldots, v_{p}\right\}$ must also have maximum perimeter among all $p$-subsets of $C$ (because (8) shows that this latter set has the same perimeter as the former set). Hence, for each $x \in C \backslash\left\{v_{1}, v_{2}, \ldots, v_{p-1}\right\}$, we have $\operatorname{PER}\left\{v_{1}, v_{2}, \ldots, v_{p}\right\} \geqslant \operatorname{PER}\left\{v_{1}, v_{2}, \ldots, v_{p-1}, x\right\}$.

Now, forget that we fixed $p$. We thus have shown that for each $p \in\{1,2, \ldots, k\}$ and each $x \in C \backslash\left\{v_{1}, v_{2}, \ldots, v_{p-1}\right\}$, we have

$$
\operatorname{PER}\left\{v_{1}, v_{2}, \ldots, v_{p}\right\} \geqslant \operatorname{PER}\left\{v_{1}, v_{2}, \ldots, v_{p-1}, x\right\} .
$$

In other words, $\left(v_{1}, v_{2}, \ldots, v_{k}\right)$ is a greedy $k$-permutation of $C$ (since $v_{1}, v_{2}, \ldots, v_{k}$ are distinct). Hence, Proposition 20 (f) (applied to $k$ and $v_{i}$ instead of $n$ and $c_{i}$ ) shows that we can find $m-k$ elements $v_{k+1}, v_{k+2}, \ldots, v_{m}$ of $C$ such that $\left(v_{1}, v_{2}, \ldots, v_{m}\right)$ is a greedy $m$-permutation of $C$. This proves Theorem 22 (since $\left.A=\left\{v_{1}, v_{2}, \ldots, v_{k}\right\}\right)$.

\section{The $\nu_{k}^{\circ}(C)$ invariants}

We shall next prove two corollaries of the above results that resemble (and, as we will later see, generalize) [2, Theorem 1] and [2, Lemma 2].

Corollary 23. Let $C \subseteq E$ be any subset. Let $m$ be a nonnegative integer. Let $k \in$ $\{1,2, \ldots, m\}$. If $\left(c_{1}, c_{2}, \ldots, c_{m}\right)$ is a greedy m-permutation of $C$, then the number

$$
w\left(c_{k}\right)+\sum_{i=1}^{k-1} d\left(c_{i}, c_{k}\right)
$$

does not depend on the choice of this m-permutation (but only depends on $k$ and on $C$ ).

Proof. Let $\left(c_{1}, c_{2}, \ldots, c_{m}\right)$ be a greedy $m$-permutation of $C$. Theorem 21 shows that for each $j \in\{0,1, \ldots, m\}$, the set $\left\{c_{1}, c_{2}, \ldots, c_{j}\right\}$ has maximum perimeter among all $j$-subsets of $C$. In other words, $F(j):=\operatorname{PER}\left\{c_{1}, c_{2}, \ldots, c_{j}\right\}$ equals the maximum possible perimeter of a $j$-subset of $C$. In particular, this number $F(j)$ depends only on $j$ and $C$. Thus, the value

$$
F(k)-F(k-1)=w\left(c_{k}\right)+\sum_{i=1}^{k-1} d\left(c_{i}, c_{k}\right)
$$

depends only on $k$ and $C$.

From now on, the number

$$
w\left(c_{k}\right)+\sum_{i=1}^{k-1} d\left(c_{i}, c_{k}\right)
$$

in Corollary 23 will be denoted by $\nu_{k}^{\circ}(C)$. 
Corollary 24. Let $C \subseteq E$ be any subset. Let $m$ be a nonnegative integer. Let $k \in$ $\{1,2, \ldots, m\}$. Let $\left(c_{1}, c_{2}, \ldots, c_{m}\right)$ be a greedy m-permutation of $C$. Let $j \in\{1,2, \ldots, k\}$. Then,

$$
\nu_{k}^{\circ}(C) \leqslant w\left(c_{j}\right)+\sum_{i \in\{1,2, \ldots, k\} \backslash\{j\}} d\left(c_{i}, c_{j}\right) .
$$

Proof. Theorem 21 shows that the set $\left\{c_{1}, c_{2}, \ldots, c_{k-1}\right\}$ has maximum perimeter among all $(k-1)$-subsets of $C$. Thus, ${ }^{7}$

$$
\operatorname{PER}\left\{c_{1}, c_{2}, \ldots, c_{k-1}\right\} \geqslant \operatorname{PER}\left\{c_{1}, c_{2}, \ldots, \widehat{c_{j}}, \ldots, c_{k}\right\}
$$

Thus

$$
\begin{aligned}
\nu_{k}^{\circ}(C) & =\operatorname{PER}\left\{c_{1}, c_{2}, \ldots, c_{k}\right\}-\operatorname{PER}\left\{c_{1}, c_{2}, \ldots, c_{k-1}\right\} \\
& \leqslant \operatorname{PER}\left\{c_{1}, c_{2}, \ldots, c_{k}\right\}-\operatorname{PER}\left\{c_{1}, c_{2}, \ldots, \widehat{c_{j}}, \ldots, c_{k}\right\} \\
& =w\left(c_{j}\right)+\sum_{i \in\{1,2, \ldots, k\} \backslash\{j\}} d\left(c_{i}, c_{j}\right) .
\end{aligned}
$$

\section{The greedoid}

Throughout this section, we assume that the set $E$ is finite.

\subsection{Defining greedoids and strong greedoids}

We shall now recall the definition of a "greedoid":

A collection ${ }^{8} \mathcal{F} \subseteq 2^{E}$ of subsets of a finite set $E$ is said to be a greedoid ${ }^{9}$ (on the ground set $E$ ) if it satisfies the following three axioms:

(i) We have $\varnothing \in \mathcal{F}$.

(ii) If $B \in \mathcal{F}$ satisfies $|B|>0$, then there exists $b \in B$ such that $B \backslash b \in \mathcal{F}$.

(iii) If $A, B \in \mathcal{F}$ satisfy $|B|=|A|+1$, then there exists $b \in B \backslash A$ such that $A \cup b \in \mathcal{F}$.

We refer to [10] for a book-length treatment of greedoids. Our above definition of a greedoid appears implicitly in [10, Section IV.1] (indeed, our axioms (i) and (iii) correspond to the conditions (1.4) and (1.6) in [10, Section IV.1], while our axioms (i) and (ii) make $(E, \mathcal{F})$ into what is called an accessible set system in [10]).

There are several classes of greedoids having additional properties besides the above three axioms. (See [10] for an overview.) Let us define one of these classes - that of "strong greedoids" (also known as "Gauss greedoids"):

A greedoid $\mathcal{F}$ on a ground set $E$ is said to be a strong greedoid if it satisfies the following axiom:

\footnotetext{
${ }^{7}$ Here, the hat over the $c_{j}$ signifies that $c_{j}$ is omitted from the list.

${ }^{8}$ The word "collection" just means "set", but will be used exclusively for sets of sets.

${ }^{9}$ More precisely, the sets in the collection are said to be the feasible sets of a greedoid. We will, however, just say that the collection is a greedoid.
} 
(iv) If $A, B \in \mathcal{F}$ satisfy $|B|=|A|+1$, then there exists some $x \in B \backslash A$ such that $A \cup x \in \mathcal{F}$ and $B \backslash x \in \mathcal{F}$.

This definition of strong greedoids appears in [6] (where the above axiom (iv) appears as property $\left.\mathrm{G}(3)^{\prime}\right)$. Note that axiom (iv) is clearly stronger than axiom (iii). The theorem in Section 2 of [6] says that strong greedoids are the same as Gauss greedoids (one of the classes of greedoids studied in [10]). See [10, Section IX.4] for further properties and characterizations of Gauss greedoids.

\subsection{The Bhargava greedoid}

The following theorem shows that a greedoid can be obtained from any ultra triple $(E, w, d)$ :

Theorem 25. Let $\mathcal{F}$ denote the collection of subsets $A \subseteq E$ that have maximum perimeter among all $|A|$-sets:

$$
\mathcal{F}=\{A \subseteq E \quad \mid \operatorname{PER}(A) \geqslant \operatorname{PER}(B) \text { for all } B \subseteq E \text { satisfying }|B|=|A|\} .
$$

Then $\mathcal{F}$ is a strong greedoid on the ground set $E$.

We call this $\mathcal{F}$ the Bhargava greedoid of the ultra triple $(E, w, d)$.

Example 26. Let $(E, w, d)$ be as in Example 2. Assume that $w(a)=0$ for all $a \in E$.

Then, the collection $\mathcal{F}$ in Theorem 25 contains $\{1,2,3\}$ and $\{1,2,3,4,5\}$ but not $\{1,2,3,5\}$.

Theorem 25 says that this collection is a strong greedoid; hence, axiom (iii) in the definition of a greedoid yields that for any $A, B \in \mathcal{F}$ satisfying $|B|=|A|+1$, there exists $b \in B \backslash A$ such that $A \cup b \in \mathcal{F}$. For example, if we pick $A=\{1,2,5\}$ and $B=\{2,3,4,5\}$, then this says that there exists $b \in\{3,4\}$ such that $\{1,2,5, b\} \in \mathcal{F}$. And indeed, $b=4$ works (though $b=3$ does not).

Example 27. Let $p=3$ and $E=\{0,1,2,3,4,5,6,12\}$. Define the distance function $d: E \times E \rightarrow \mathbb{R}$ as in Example 4. Set $w(e)=0$ for all $e \in E$.

Then, the collection $\mathcal{F}$ in Theorem 25 contains $\{0,1,2\}$ and $\{0,1,2,3\}$ and $\{0,1,2,6\}$ and $\{0,1,2,4,5,6,12\}$ but not $\{0,1,2,3,6\}$ and not $\{0,1,2,3,4,5,12\}$.

For readers familiar with the alternative description of greedoids as hereditary languages (see, e.g., [10, Section IV.1]), we note in passing that the language corresponding to the greedoid $\mathcal{F}$ in Theorem 25 is precisely the set of greedy $m$-permutations for $m \geqslant 0$. This observation will not be used in what follows, but helps illuminate the proofs.

Our proof of Theorem 25 will rely on the following lemma (inspired by [11, Theorem $3.2])$ :

Lemma 28. Let $A$ and $B$ be two subsets of $E$ such that $|B|=|A|+1$.

Then, there exists $a u \in B \backslash A$ satisfying

$$
\operatorname{PER}(B \backslash u)+\operatorname{PER}(A \cup u) \geqslant \operatorname{PER}(A)+\operatorname{PER}(B) .
$$


Proof of Lemma 28. Let $k=|A|$; thus, $|B|=|A|+1=k+1$. Let $\left(a_{1}, a_{2}, \ldots, a_{k}\right)$ be a list of all $k$ elements of $A$ (with no repetitions).

We define a list $\left(b_{1}, b_{2}, \ldots, b_{k}\right)$ of $k$ elements of $B$ recursively as follows: For each $i=1,2, \ldots, k$, we let $b_{i}$ be a projection of $a_{i}$ onto $B \backslash\left\{b_{1}, b_{2}, \ldots, b_{i-1}\right\}$ (assuming that $b_{1}, b_{2}, \ldots, b_{i-1}$ have already been constructed). ${ }^{10}$ Thus, $b_{1}, b_{2}, \ldots, b_{k}$ are $k$ distinct $^{11}$ elements of $B$. Thus, $\left\{b_{1}, b_{2}, \ldots, b_{k}\right\}$ is a $k$-element subset of $B$. Hence, its complement $B \backslash\left\{b_{1}, b_{2}, \ldots, b_{k}\right\}$ has size $|B|-k=1$ (since $|B|=k+1$ ). In other words, there is a unique element $u \in B \backslash\left\{b_{1}, b_{2}, \ldots, b_{k}\right\}$. Consider this $u$. Hence, $B \backslash\left\{b_{1}, b_{2}, \ldots, b_{k}\right\}=\{u\}$, so that $B \backslash u=\left\{b_{1}, b_{2}, \ldots, b_{k}\right\}$. From $u \in B \backslash\left\{b_{1}, b_{2}, \ldots, b_{k}\right\}$, we obtain $u \notin\left\{b_{1}, b_{2}, \ldots, b_{k}\right\}$.

We have $u \notin A$.

[Proof: Assume the contrary. Thus, $u \in A=\left\{a_{1}, a_{2}, \ldots, a_{k}\right\}$. Hence, $u=a_{i}$ for some $i \in\{1,2, \ldots, k\}$. Consider this $i$. But $a_{i}=u \in B \backslash\left\{b_{1}, b_{2}, \ldots, b_{k}\right\} \subseteq B \backslash\left\{b_{1}, b_{2}, \ldots, b_{i-1}\right\}$. Hence, Lemma 13 (a) (applied to $B \backslash\left\{b_{1}, b_{2}, \ldots, b_{i-1}\right\}, a_{i}$ and $b_{i}$ instead of $C, v$ and $u$ ) yields $b_{i}=a_{i}$ (because $b_{i}$ is defined as a projection of $a_{i}$ onto $B \backslash\left\{b_{1}, b_{2}, \ldots, b_{i-1}\right\}$ ). Hence, $u=a_{i}=b_{i}$, which contradicts $u \notin\left\{b_{1}, b_{2}, \ldots, b_{k}\right\}$. This contradiction shows that our assumption was false. Hence, $u \notin A$ is proven.]

Combining $u \in B$ with $u \notin A$, we find $u \in B \backslash A$.

For each $i \in\{1,2, \ldots, k\}$, we have

$$
d\left(a_{i}, u\right) \geqslant d\left(b_{i}, u\right)
$$

[Proof: Let $i \in\{1,2, \ldots, k\}$. Then, from $u \notin\left\{b_{1}, b_{2}, \ldots, b_{k}\right\}$, we obtain $u \neq b_{i}$. Also, $u \in B \backslash\left\{b_{1}, b_{2}, \ldots, b_{k}\right\} \subseteq B \backslash\left\{b_{1}, b_{2}, \ldots, b_{i-1}\right\}$, whereas $b_{i}$ is a projection of $a_{i}$ onto $B \backslash\left\{b_{1}, b_{2}, \ldots, b_{i-1}\right\}$. Hence, Lemma 13 (c) (applied to $B \backslash\left\{b_{1}, b_{2}, \ldots, b_{i-1}\right\}, a_{i}, b_{i}$ and $u$ instead of $C, v, u$ and $x$ ) shows that $d\left(b_{i}, u\right) \leqslant d\left(a_{i}, u\right)$ (since $\left.u \neq b_{i}\right)$. This proves (11).]

From $u \in B$, we obtain

$$
\begin{aligned}
\operatorname{PER}(B \backslash u) & =\operatorname{PER}(B)-w(u)-\sum_{b \in B \backslash u} d(b, u) \\
& =\operatorname{PER}(B)-w(u)-\sum_{i=1}^{k} d\left(b_{i}, u\right)
\end{aligned}
$$

(since $B \backslash u=\left\{b_{1}, b_{2}, \ldots, b_{k}\right\}$ and since $b_{1}, b_{2}, \ldots, b_{k}$ are distinct).

From $u \notin A$, we obtain

$$
\begin{aligned}
\operatorname{PER}(A \cup u) & =\operatorname{PER}(A)+w(u)+\sum_{a \in A} d(a, u) \\
& =\operatorname{PER}(A)+w(u)+\sum_{i=1}^{k} d\left(a_{i}, u\right)
\end{aligned}
$$

(since $A=\left\{a_{1}, a_{2}, \ldots, a_{k}\right\}$, and since $a_{1}, a_{2}, \ldots, a_{k}$ are distinct)

\footnotetext{
${ }^{10}$ Thus, in particular, $b_{1}$ is a projection of $a_{1}$ onto $B$.

${ }^{11}$ The distinctness of $b_{1}, b_{2}, \ldots, b_{k}$ follows from $b_{i} \in B \backslash\left\{b_{1}, b_{2}, \ldots, b_{i-1}\right\}$.
} 


$$
\geqslant \operatorname{PER}(A)+w(u)+\sum_{i=1}^{k} d\left(b_{i}, u\right) \quad(\text { by }(11)) .
$$

Adding this inequality to the equality (12), we obtain

$$
\begin{aligned}
\operatorname{PER}(B \backslash u)+\operatorname{PER}(A \cup u) & \geqslant \operatorname{PER}(B)+\operatorname{PER}(A) \\
& =\operatorname{PER}(A)+\operatorname{PER}(B) .
\end{aligned}
$$

This is precisely the inequality (10).

Thus, we have found a $u \in B \backslash A$ satisfying (10). Hence, such a $u$ exists. This proves Lemma 28.

Proof of Theorem 25. We only need to prove the two axioms (i) and (ii) from the definition of a greedoid and the axiom (iv) from the definition of a strong greedoid (because axiom (iii) will follow from axiom (iv)).

Axiom (i) is obvious.

Next, let us prove axiom (iv). So let $A, B \in \mathcal{F}$ be such that $|B|=|A|+1$. We must prove that there exists some $x \in B \backslash A$ such that $A \cup x \in \mathcal{F}$ and $B \backslash x \in \mathcal{F}$.

Lemma 28 shows that there exists a $u \in B \backslash A$ satisfying (10). Consider this $u$.

Let $k=|A|$; thus, $|B|=|A|+1=k+1$. But $u \in B \backslash A \subseteq B$, so that $|B \backslash u|=$ $|B|-1=k$ (since $|B|=k+1$ ). Thus, $B \backslash u$ is a $k$-set. But $A$ is a $k$-set in $\mathcal{F}$, and thus has the largest perimeter among all $k$-sets. Hence, $\operatorname{PER}(A) \geqslant \operatorname{PER}(B \backslash u)$.

Furthermore, $u \in B \backslash A$, thus $u \notin A$, so that $|A \cup u|=|A|+1=k+1$. Hence, $A \cup u$ is a $(k+1)$-set. But $B$ is a $(k+1)$-set in $\mathcal{F}$, and thus has the largest perimeter among all $(k+1)$-sets. Hence, $\operatorname{PER}(B) \geqslant \operatorname{PER}(A \cup u)$. Adding this inequality to $\operatorname{PER}(A) \geqslant$ $\operatorname{PER}(B \backslash u)$, we obtain

$$
\operatorname{PER}(A)+\operatorname{PER}(B) \geqslant \operatorname{PER}(B \backslash u)+\operatorname{PER}(A \cup u) .
$$

Contrasting this inequality with the opposite inequality (10) (which, as we know, is satisfied), we conclude that it must be an equality. Hence, both inequalities $\operatorname{PER}(A) \geqslant$ $\operatorname{PER}(B \backslash u)$ and PER $(B) \geqslant \operatorname{PER}(A \cup u)$ (which we added to obtain it) must be equalities as well. In other words, $\operatorname{PER}(A)=\operatorname{PER}(B \backslash u)$ and $\operatorname{PER}(B)=\operatorname{PER}(A \cup u)$. Hence, $B \backslash u$ is a $k$-set of maximum perimeter (since $A$ is a $k$-set of maximum perimeter, but $\operatorname{PER}(A)=\operatorname{PER}(B \backslash u)$ ), and thus belongs to $\mathcal{F}$; in other words, $B \backslash u \in \mathcal{F}$. Likewise, from the other inequality, we obtain $A \cup u \in \mathcal{F}$. Hence, there exists some $x \in B \backslash A$ such that $A \cup x \in \mathcal{F}$ and $B \backslash x \in \mathcal{F}$ (namely, $x=u$ ). Thus, axiom (iv) is proven.

Let us now prove axiom (ii). So let $B \in \mathcal{F}$ satisfy $|B|>0$. Then, $|B|-1 \in$ $\{0,1, \ldots,|E|\}$. Hence, there exists at least one $(|B|-1)$-subset of $E$. Since $E$ is finite, we can thus find a $(|B|-1)$-subset of $E$ having maximum perimeter (among all $(|B|-1)$ subsets of $E$ ). Choose such a subset, and denote it by $A$. Thus, $A \in \mathcal{F}$ (by the definition of $\mathcal{F}$, since $A$ has maximum perimeter) and $|B|=|A|+1$ (since $|A|$ is a $(|B|-1)$-subset). Hence, axiom (iv) (which we have already proved) shows that there exists some $x \in B \backslash A$ such that $A \cup x \in \mathcal{F}$ and $B \backslash x \in \mathcal{F}$. Consider this $x$. Thus, $x \in B \backslash A \subseteq B$. Hence, there exists $b \in B$ such that $B \backslash b \in \mathcal{F}$ (namely, $b=x$ ). This proves axiom (ii).

This shows that $\mathcal{F}$ is a strong greedoid. 
We now know that the Bhargava greedoid $\mathcal{F}$ of an ultra triple is a strong greedoid. It is natural to inquire which other known classes of greedoids $\mathcal{F}$ belongs to. However, for many of these classes (including interval greedoids), the answer is negative, because $\mathcal{F}$ is (in general) not a transposition greedoid. We refer to [10, Chapter $\mathrm{X}]$ for the definition of transposition greedoids (and for why many classes of greedoids are subclasses of transposition greedoids); let us merely remark that the Bhargava greedoid $\mathcal{F}$ fails to be a transposition greedoid in Example 27, since the transposition property [10, (1.1) in Section X.1] is violated for $A=\{0,1,2\}, x=3, y=6$ and $B=\{4,5,12\}$.

The Bhargava greedoid $\mathcal{F}$ also fails to be a transversal greedoid in the sense of $[5]^{12}$. Indeed, the ultra triple $(E, w, d)$ constructed in Example 4 for $p=2$ and $E=\{1,2,3,4\}$ provides a counterexample ${ }^{13}$.

Another class of greedoids that the Bhargava greedoid $\mathcal{F}$ does not belong to is that of twisted matroids ([10, Section IV.2.18]). Indeed, [9, Proposition 3.1] shows that every twisted matroid is a $\Delta$-matroid (see $[9$, Section 2.4] for a definition of the latter concept); but $\mathcal{F}$ is not in general a $\Delta$-matroid ${ }^{14}$.

In [8], it is shown that the Bhargava greedoid $\mathcal{F}$ is a Gaussian elimination greedoid (see [10, Sections IV.2 and IX.4] for this concept).

Question 29. Is $\mathcal{F}$ a linking greedoid? (This is yet another subclass of Gauss greedoids, and can in some sense be understood as "Gaussian elimination greedoids over the field with one element"; see again [10, Sections IV.2 and IX.4].)

\section{The matroid}

Throughout this section, we assume that the set $E$ is finite.

\subsection{Defining matroids}

We shall now recall one of the many definitions of a matroid. Namely, if $E$ is a finite set, $k$ is a nonnegative integer, and $\mathcal{B}$ is a collection of $k$-subsets of $E$, then we say that $\mathcal{B}$ is the collection of bases of a matroid if and only if $\mathcal{B}$ is nonempty and satisfies the following axiom: ${ }^{15}$

- For any two $k$-subsets $B_{1}, B_{2} \in \mathcal{B}$ and any $x \in B_{1} \backslash B_{2}$, there exists a $y \in B_{2} \backslash B_{1}$ such that $B_{1} \cup y \backslash x \in \mathcal{B}$.

\footnotetext{
${ }^{12}$ Transversal greedoids are the same as medieval marriage greedoids in the sense of [10, Section IV.2.14].

${ }^{13}$ The easiest way to check this is to observe that it violates the condition (M3) ${ }^{\dagger}$ from [5, Theorem 2.1]. (Note that there is a typo in $\left[5\right.$, Theorem 2.1]: In Condition $(\mathrm{M} 3)^{\dagger}$, replace " $Z \neq \varnothing$ " by " $X \neq \varnothing$ ".)

${ }^{14}$ For an example, use the ultra triple $(E, w, d)$ constructed in Example 4 for $p=2$ and $E=\{1,2,4,8\}$. Here, the axiom defining a $\Delta$-matroid fails for $X=\{1,2,4,8\}, Y=\varnothing$ and $x=1$.

${ }^{15}$ This axiom is condition (1.4) in [10, Section II.1]. See [10, Theorem II.1.1] for a proof of its equivalence to other definitions of a matroid. See also [13] for much more about matroids.
} 


\subsection{Matroids from strong greedoids}

We now get to the main result of this section:

Theorem 30. The Bhargava greedoid $\mathcal{F}$ has the following property: Fix a nonnegative integer $k \leqslant|E|$. All sets $A \in \mathcal{F}$ having size $k$ form the collection of bases of a matroid.

Not all greedoids enjoy this property. For example, if $\{a, b, c, d\}$ is a poset with two inequalities $a<b$ and $c<d$, then the greedoid of lower ideals of this poset contains the subsets $\{a, b\}$ and $\{c, d\}$, but $a$ in the set $\{a, b\}$ cannot be replaced by any of $c$ and $d$.

However, all strong greedoids (i.e., Gauss greedoids) enjoy this property:

Theorem 31. Let $\mathcal{F}$ be a strong greedoid on the ground set $E$. Let $B_{1} \in \mathcal{F}$ and $B_{2} \in \mathcal{F}$ satisfy $\left|B_{1}\right|=\left|B_{2}\right|$. Let $x \in B_{1} \backslash B_{2}$. Then, there exists some $y \in B_{2} \backslash B_{1}$ such that $B_{1} \cup y \backslash x \in \mathcal{F}$.

Theorem 31 is (implicitly) proven in the third paragraph of [6, Proof of the Theorem]. For the sake of completeness, we shall present this proof in a slightly modified form below. First, we need two lemmas about greedoids:

Lemma 32. Let $\mathcal{F}$ be a greedoid on the ground set $E$. Let $A, B \in \mathcal{F}$ satisfy $|B|>|A|$. Then, there exists some $b \in B \backslash A$ such that $A \cup b \in \mathcal{F}$.

Proof of Lemma 32. A nice set will mean a subset $C$ of $B$ such that $|C|>|A|$ and $C \in \mathcal{F}$. There exists at least one nice set (namely, $B$ is a nice set). Thus, there exists a nice set of smallest possible size. Let $D$ be such a set. Thus, $D$ is a subset of $B$ such that $|D|>|A|$ and $D \in \mathcal{F}$ (since $D$ is a nice set). Hence, $|D|>|A| \geqslant 0$. Thus, axiom (ii) in the definition of a greedoid (applied to $D$ instead of $B$ ) shows that there exists a $b \in D$ such that $D \backslash b \in \mathcal{F}$. Pick such a $b$ and denote it by $d$. Thus, $d \in D$ and $D \backslash d \in \mathcal{F}$. Note that $D \backslash d$ is a subset of $B$ (since $D$ is), and has smaller size than $D$ (since $d \in D$ ). Hence, if we had $|D \backslash d|>|A|$, then $D \backslash d$ would be a nice set of smaller size than $D$; but this would contradict the fact that $D$ is a nice set of smallest possible size. Thus, we must have $|D \backslash d| \leqslant|A|$. Since $d \in D$, we have $|D \backslash d|=|D|-1$, so that $|D|-1=|D \backslash d| \leqslant|A|$, and therefore $|D| \leqslant|A|+1$. Combining this with $|D|>|A|$, we obtain $|D|=|A|+1$. Hence, axiom (iii) in the definition of a greedoid (applied to $D$ instead of $B$ ) shows that there exists a $b \in D \backslash A$ such that $A \cup b \in \mathcal{F}$. Consider this $b$. We have $b \in D \backslash A \subseteq B \backslash A$ (since $D \subseteq B$ ). Thus, we have found a $b \in B \backslash A$ such that $A \cup b \in \mathcal{F}$. This proves Lemma 32 .

Lemma 33. Let $\mathcal{F}$ be a strong greedoid on the ground set $E$. Let $D$ be a subset of $E$, and let $x, y, z$ be three elements of $E \backslash D$. Assume that $D \cup\{x, z\} \in \mathcal{F}$ and $D \cup y \in \mathcal{F}$ and $D \cup z \notin \mathcal{F}$. Then, we have $D \cup\{y, z\} \in \mathcal{F}$.

Proof of Lemma 33. We have $D \cup\{x, z\} \neq D \cup z$ (since $D \cup\{x, z\} \in \mathcal{F}$ but $D \cup z \notin \mathcal{F}$ ). Hence, $x \neq z$. Furthermore, none of the elements $x, y, z$ belongs to $D$ (since they all belong to $E \backslash D)$. Hence, $|D \cup\{x, z\}|=|D \cup y|+1$ (since $x \neq z$ ). Consequently, axiom (iv) in the definition of a strong greedoid (applied to $A=D \cup y$ and $B=D \cup\{x, z\}$ ) 
yields that there exists some $t \in(D \cup\{x, z\}) \backslash(D \cup y)$ such that $(D \cup y) \cup t \in \mathcal{F}$ and $(D \cup\{x, z\}) \backslash t \in \mathcal{F}$. Consider this $t$.

Combining $x \neq z$ with $x \notin D$, we obtain $x \notin D \cup z$. If we had $t=x$, then we would have $\underbrace{(D \cup\{x, z\})}_{=(D \cup z) \cup x} \backslash \underbrace{t}_{=x}=(D \cup z) \cup x \backslash x=D \cup z$ (since $x \notin D \cup z$ ) and therefore $D \cup z=(D \cup\{x, z\}) \backslash t \in \mathcal{F}$, which would contradict $D \cup z \notin \mathcal{F}$. Hence, we must have $t \neq x$.

We have $t \in(D \cup\{x, z\}) \backslash(D \cup y) \subseteq\{x, z\}$, so that either $t=x$ or $t=z$. Thus, $t=z$ (since $t \neq x$ ). Hence, $z=t$, so that $D \cup\{y, z\}=(D \cup y) \cup \underbrace{z}_{=t}=(D \cup y) \cup t \in \mathcal{F}$.

Proof of Theorem 31. From $x \in B_{1} \backslash B_{2}$, we obtain $x \in B_{1}$ and $x \notin B_{2}$. Hence, $\left|B_{1} \backslash x\right|=$ $\left|B_{1}\right|-1$.

A free set will mean a subset $A$ of $B_{1} \backslash x$ such that $A \in \mathcal{F}$. Clearly, a free set exists (indeed, $\varnothing$ is a free set, since axiom (i) in the definition of a greedoid yields $\varnothing \in \mathcal{F}$ ). Hence, there exists a free set of largest size. Pick such a free set, and denote it by $A$. Thus, $A$ is a subset of $B_{1} \backslash x$ and satisfies $A \in \mathcal{F}$ (since $A$ is a free set). Since $A$ is a subset of $B_{1} \backslash x$, we have $|A| \leqslant\left|B_{1} \backslash x\right|=\left|B_{1}\right|-1<\left|B_{1}\right|=\left|B_{2}\right|$. Thus, Lemma 32 (applied to $B=B_{2}$ ) yields that there exists some $b \in B_{2} \backslash A$ such that $A \cup b \in \mathcal{F}$. Consider this $b$, and denote it by $y$. Thus, $y \in B_{2} \backslash A$ and $A \cup y \in \mathcal{F}$.

Next, we claim that $A \cup x \in \mathcal{F}$.

[Proof: Assume the contrary. Thus, $A \cup x \notin \mathcal{F}$. Recall that $|A|<\left|B_{1}\right|$. Thus, Lemma 32 (applied to $B=B_{1}$ ) yields that there exists some $b \in B_{1} \backslash A$ such that $A \cup b \in \mathcal{F}$. Consider this $b$. Clearly, $b \notin A$. We have $A \cup b \neq A \cup x$ (since $A \cup b \in \mathcal{F}$ but $A \cup x \notin \mathcal{F}$ ), and thus $b \neq x$. Hence, $b \in B_{1} \backslash x$ (since $b \in B_{1} \backslash A \subseteq B_{1}$ ). Clearly, the set $A \cup b$ has larger size than $A$ (since $b \notin A$ ). Now, $A \cup b$ is a subset of $B_{1} \backslash x$ (since $A \subseteq B_{1} \backslash x$ and $b \in B_{1} \backslash x$ ), and thus is a free set (since $A \cup b \in \mathcal{F}$ ) of larger size than $A$. This contradicts the fact that $A$ is a free set of largest size. This contradiction shows that our assumption was wrong. Hence, we have shown that $A \cup x \in \mathcal{F}$.]

From $y \in B_{2} \backslash A$, we obtain $y \in B_{2}$ and $y \notin A$. Hence, the set $A \cup y$ has larger size than $A$ (since $y \notin A$ ). If we had $y \in B_{1} \backslash x$, then $A \cup y$ would be a subset of $B_{1} \backslash x$ (since $A \subseteq B_{1} \backslash x$ ), and therefore $A \cup y$ would be a free set (since $A \cup y \in \mathcal{F}$ ) of larger size than $A$; this would contradict the fact that $A$ is a free set of largest size. Hence, $y \notin B_{1} \backslash x$. Since $y \neq x$ (because $y \in B_{2}$ but $x \notin B_{2}$ ), we thus obtain $y \notin B_{1}$. Hence, $y \in B_{2} \backslash B_{1}$ (since $y \in B_{2}$ ).

Thus, if $B_{1} \cup y \backslash x \in \mathcal{F}$, then Theorem 31 is proven. Hence, for the sake of contradiction, we assume that $B_{1} \cup y \backslash x \notin \mathcal{F}$.

A useful set will mean a set $C \subseteq E$ such that $A \subseteq C \subseteq B_{1} \backslash x$ and $C \cup x \in \mathcal{F}$ and $C \cup y \in \mathcal{F}$. The set $A$ is a useful set (since $A \subseteq A \subseteq B_{1} \backslash x$ and $A \cup x \in \mathcal{F}$ and $A \cup y \in \mathcal{F}$ ). Hence, there exists a useful set. Thus, there exists a useful set of maximum size. Let $D$ be such a set. Thus, $D$ is a useful set; that is, $D \subseteq E$ and $A \subseteq D \subseteq B_{1} \backslash x$ and $D \cup x \in \mathcal{F}$ and $D \cup y \in \mathcal{F}$.

We have $y \neq x$ and thus $\left(B_{1} \backslash x\right) \cup y=B_{1} \cup y \backslash x \notin \mathcal{F}$. Hence, the set $B_{1} \backslash x$ is not a useful set. Thus, $D \neq B_{1} \backslash x$ (since $D$ is a useful set). Therefore, $D$ is a proper subset 
of $B_{1} \backslash x$ (since $D \subseteq B_{1} \backslash x$ ). Hence, $|D|<\left|B_{1} \backslash x\right|=\left|B_{1}\right|-1$ (since $x \in B_{1}$ ). Thus, $|D|+1<\left|B_{1}\right|$, so that $|D \cup x| \leqslant|D|+1<\left|B_{1}\right|$.

The two sets $D \cup x$ and $B_{1}$ belong to $\mathcal{F}$ and satisfy $|D \cup x|<\left|B_{1}\right|$. Hence, Lemma 32 (applied to $D \cup x$ and $B_{1}$ instead of $A$ and $B$ ) yields that there exists some $b \in B_{1} \backslash(D \cup x)$ such that $D \cup x \cup b \in \mathcal{F}$. Consider this $b$, and denote it by $z$. Thus, $z \in B_{1} \backslash(D \cup x)$ and $D \cup x \cup z \in \mathcal{F}$. Hence, $D \cup\{x, z\}=D \cup x \cup z \in \mathcal{F}$. Furthermore, $x \notin D$ (since $D \subseteq B_{1} \backslash x$ ) and $y \notin D$ (since $y \notin B_{1}$ but $\left.D \subseteq B_{1} \backslash x \subseteq B_{1}\right)$ and $z \notin D$ (since $z \in B_{1} \backslash(D \cup x)$ and thus $z \notin D \cup x$, so that $z \notin D)$. Hence, all of $x, y, z$ are elements of $E \backslash D$.

The set $D \cup z$ has larger size than $D$ (since $z \notin D$ ), and thus has larger size than $A$ (since $A \subseteq D$ entails $|A| \leqslant|D|)$. Combining $D \subseteq B_{1} \backslash x$ and $z \in B_{1} \backslash(D \cup x) \subseteq B_{1} \backslash x$, we obtain $D \cup z \subseteq B_{1} \backslash x$. Hence, if we had $D \cup z \in \mathcal{F}$, then $D \cup z$ would be a free set of larger size than $A$. This would contradict the fact that $A$ is a free set of largest size. Hence, $D \cup z \notin \mathcal{F}$. Thus, Lemma 33 shows that $D \cup\{y, z\} \in \mathcal{F}$. Now, the set $D \cup z$ has larger size than $D$ and satisfies $A \subseteq D \cup z \subseteq B_{1} \backslash x$ (since $A \subseteq D \subseteq D \cup z$ and $D \cup z \subseteq B_{1} \backslash x$ ) and $(D \cup z) \cup x=D \cup\{x, z\} \in \mathcal{F}$ and $(D \cup z) \cup y=D \cup\{y, z\} \in \mathcal{F}$. Hence, $D \cup z$ is a useful set of larger size than $D$. This contradicts the fact that $D$ is a useful set of maximum size. This contradiction shows that our assumption (that $B_{1} \cup y \backslash x \notin \mathcal{F}$ ) was wrong. Hence, $B_{1} \cup y \backslash x \in \mathcal{F}$. This proves Theorem 31 .

We note that the condition " $\left|B_{1}\right|=\left|B_{2}\right|$ " in Theorem 31 could be replaced by the weaker condition " $\left|B_{1}\right| \leqslant\left|B_{2}\right|$ ". Indeed, our proof of Theorem 31 only used the latter condition.

Proof of Theorem 30. The assumption $k \leqslant|E|$ shows that there exist $k$-sets. Some of them have maximum perimeter (since $E$ is finite). Hence, the collection of all sets $A \in \mathcal{F}$ having size $k$ is nonempty.

Theorem 25 shows that $\mathcal{F}$ is a strong greedoid. Hence, Theorem 31 shows that for any two sets $B_{1} \in \mathcal{F}$ and $B_{2} \in \mathcal{F}$ satisfying $\left|B_{1}\right|=\left|B_{2}\right|$ and for any $x \in B_{1} \backslash B_{2}$, there exists some $y \in B_{2} \backslash B_{1}$ such that $B_{1} \cup y \backslash x \in \mathcal{F}$. This yields that all sets $A \in \mathcal{F}$ having size $k$ form the collection of bases of a matroid (because if $B_{1}$ and $B_{2}$ are two sets of size $k$, and if $x \in B_{1} \backslash B_{2}$ and $y \in B_{2} \backslash B_{1}$, then $B_{1} \cup y \backslash x$ is a set of size $k$ as well). This is precisely the claim of Theorem 30 .

\section{Greedy subsequences}

We shall now study a slight variation of the notion of greedy $m$-permutations, in which we allow picking the same point multiple times. This requires us to consider distances of the form $d(a, a)$, which our definition of ultra triple does not support. Thus, we begin by introducing a somewhat stronger concept, that of "full ultra triples".

\subsection{Full ultra triples}

Consider again a set $E$. 
As before, we assume that a function $w: E \rightarrow \mathbb{R}$ is given, which assigns a weight $w(a)$ to each point $a \in E$.

Assume further that we are given a function $d: E \times E \rightarrow \mathbb{R}$, which we will call the distance function. Thus, any two points $a, b \in E$ have a real-valued distance $d(a, b)$. We assume that this distance function has the following properties:

- It is symmetric: that is, $d(a, b)=d(b, a)$ for any $a, b \in E$.

- It satisfies the following inequality:

$$
d(a, b) \leqslant \max \{d(a, c), d(b, c)\}
$$

for any $a, b, c \in E$.

(Again, (13) is just the ultrametric triangle inequality; but keep in mind that $d(a, a)$ can be nonzero and even negative, unlike in a metric space.)

Such a structure $(E, w, d)$ will be called a full ultra triple. Thus, the notion of a full ultra triple differs from that of an ultra triple in that the distance function $d$ is defined on $E \times E$ rather than on $E \underline{\times} E$ (so that the distances $d(a, b)$ are defined for $a=b$ as well).

Example 34. Consider the situation of Example 9, but now define a map $d: E \times E \rightarrow \mathbb{R}$ by the same formula that was used to define the map $d: E \underline{\times} E \rightarrow \mathbb{R}$ in Example 9. Then, $(E, w, d)$ is a full ultra triple.

It is immediately clear that if $(E, w, d)$ is a full ultra triple, then $(E, w, \underline{d})$ is an ultra triple, where $\underline{d}: E \underline{\times} E \rightarrow \mathbb{R}$ is the restriction of $d$ to the subset $E \times E$ of $E \times E$. In other words, any full ultra triple $(E, w, d)$ becomes an ultra triple if we restrict the distance function $d$ to $E \underline{\times} E$ (that is, if we forget the distances $d(a, a)$ between each point and itself). Thus, any concept that was defined for ultra triples (e.g., the concept of a greedy $m$-permutation) is automatically defined for any full ultra triple $(E, w, d)$ as well (just apply it to $(E, w, \underline{d}))$, and any proposition that has been proven for all ultra triples can be applied to all full ultra triples.

Conversely, we can often - but not always - transform an ultra triple into a full ultra triple as follows:

Remark 35. Let $(E, w, d)$ be an ultra triple.

Fix an $N \in \mathbb{R}$ with the property that

$$
N \leqslant d(a, b) \quad \text { for all }(a, b) \in E \underline{x} E .
$$

(Such an $N$ always exists when $E$ is finite.)

Define a map $\bar{d}: E \times E \rightarrow \mathbb{R}$ by setting

$$
\bar{d}(a, b)=\left\{\begin{array}{ll}
d(a, b), & \text { if } a \neq b ; \\
N, & \text { if } a=b
\end{array} \quad \text { for all } a, b \in E .\right.
$$

Then, $(E, w, \bar{d})$ is a full ultra triple. This full ultra triple extends the original ultra triple $(E, w, d)$ (in the sense that the distance function $d$ of the latter is a restriction of $\bar{d}$ ). 
The proof of Remark 35 is a refreshing (if very simple) exercise that we leave to the reader.

Example 36. For this example, we fix a prime number $p$ and a subset $E$ of $\mathbb{Z}$. Define an ultra triple $(E, w, d)$ as in Example 4.

Then, $0 \leqslant d(a, b)$ for all $(a, b) \in E \times E$. Hence, we can define a map $\bar{d}: E \times E \rightarrow \mathbb{R}$ as in Remark 35 (by setting $N=0$ ), and obtain a full ultra triple $(E, w, \bar{d})$ that extends our ultra triple $(E, w, d)$.

Example 37. Let us see an example where the construction in Remark 35 does not work.

For this example, we fix a prime number $p$ and a subset $E$ of $\mathbb{Z}$. Define an ultra triple $\left(E, w, d^{\prime}\right)$ as in Example 5 .

If $E$ is infinite, then there exists no $N \in \mathbb{R}$ with the property that $N \leqslant d^{\prime}(a, b)$ for all $(a, b) \in E \times E$. (Indeed, for each $m \in \mathbb{N}$, there exist two distinct elements $a$ and $b$ of $E$ satisfying $a \equiv b \bmod p^{m}$ and therefore $d^{\prime}(a, b) \leqslant-m$.) Hence, we cannot define $\bar{d}$ as in Remark 35. ${ }^{16}$

It is tempting to try fixing this issue by setting $d^{\prime}(a, a)=-\infty$ for all $a \in E$. However, this would require a generalization of the notion of a full ultra triple, allowing distances to be $-\infty$; this, in turn, would cause some complications in our proofs ${ }^{17}$. Thus we are not making this generalization.

Note that every full ultra triple $(E, w, d)$ satisfies

$$
d(a, a) \leqslant d(a, c) \quad \text { for all } a, c \in E .
$$

In fact, this follows by substituting $b=a$ in (13).

\subsection{Further definitions}

For the rest of Section 8, we shall fix a full ultra triple $(E, w, d)$.

If $B \subseteq E$ and if $m$ is a nonnegative integer, then an $m$-subsequence of $B$ shall mean an $m$-tuple of elements of $B$ (not necessarily distinct).

If $\mathbf{a}=\left(a_{1}, a_{2}, \ldots, a_{m}\right) \in E^{m}$ is any $m$-tuple, then we define its perimeter PER (a) as

$$
\operatorname{PER}(\mathbf{a}):=\sum_{k=1}^{m} w\left(a_{k}\right)+\sum_{1 \leqslant i<j \leqslant m} d\left(a_{i}, a_{j}\right) .
$$

This generalizes the perimeter of an $m$-set; in fact, if the entries of the $m$-tuple $\mathbf{a}=$ $\left(a_{1}, a_{2}, \ldots, a_{m}\right) \in E^{m}$ are distinct, then $\operatorname{PER}(\mathbf{a})=\operatorname{PER}\left\{a_{1}, a_{2}, \ldots, a_{m}\right\}$.

If an $m$-tuple $\mathbf{a} \in E^{m}$ is a permutation of an $m$-tuple $\mathbf{b} \in E^{m}$, then $\operatorname{PER}(\mathbf{a})=$ $\operatorname{PER}(\mathbf{b})$. (This follows from the requirement $d(a, b)=d(b, a)$ on our distance function.)

\footnotetext{
${ }^{16}$ This does not mean that the ultra triple $\left(E, w, d^{\prime}\right)$ cannot be obtained by restricting a full ultra triple. Sometimes it can (for example, when $E=\left\{p^{0}, p^{1}, p^{2}, \ldots\right\}$ ); sometimes it cannot (for example, when $E=\mathbb{Z}$ ).

${ }^{17}$ In our proofs, we used the fact that if a sum of finitely many inequalities between real numbers is an equality, then each of the inequalities being summed must itself be an equality. (In other words: If $\left(a_{i}\right)_{i \in I}$ and $\left(b_{i}\right)_{i \in I}$ are two finite families of reals satisfying $a_{i} \geqslant b_{i}$ for all $i \in I$ and $\sum_{i \in I} a_{i}=\sum_{i \in I} b_{i}$, then $a_{i}=b_{i}$ for all $i \in I$.) This is no longer true if we allow $-\infty$ as an addend.
} 
Definition 38. Let $C \subseteq E$ be any subset, and let $m$ be a nonnegative integer.

A greedy $m$-subsequence of $C$ is an $m$-subsequence $\left(c_{1}, c_{2}, \ldots, c_{m}\right)$ of $C$ such that for each $i \in\{1,2, \ldots, m\}$ and each $x \in C$, we have

$$
\operatorname{PER}\left(c_{1}, c_{2}, \ldots, c_{i}\right) \geqslant \operatorname{PER}\left(c_{1}, c_{2}, \ldots, c_{i-1}, x\right) .
$$

Thus, this notion differs from the notion of a greedy $m$-permutation in two aspects: A greedy $m$-subsequence is allowed to have equal entries, and the inequality (16) is required to hold for all $x \in C$ (rather than only for $x \in C \backslash\left\{c_{1}, c_{2}, \ldots, c_{i-1}\right\}$ ). Thus, greedy $m$-subsequences are like greedy $m$-permutations except that we are sampling with replacement.

\subsection{Main analogues}

We can now state the following analogues of Theorem 21, Theorem 22, Corollary 23 and Corollary 24, respectively:

Theorem 39. Let $C \subseteq E$ be any subset, and let $m$ be a nonnegative integer.

Let $\left(c_{1}, c_{2}, \ldots, c_{m}\right)$ be any greedy $m$-subsequence of $C$.

Then, for each $k \in\{0,1, \ldots, m\}$, the $k$-subsequence $\left(c_{1}, c_{2}, \ldots, c_{k}\right)$ has maximum perimeter among all $k$-subsequences of $C$.

Theorem 40. Let $C \subseteq E$ be any finite nonempty subset, and let $m$ be a nonnegative integer. Let $k \in\{0,1, \ldots, m\}$.

Let $\mathbf{a}$ be any $k$-subsequence of $C$ with maximum perimeter. Then, there exists a greedy m-subsequence $\left(c_{1}, c_{2}, \ldots, c_{m}\right)$ of $C$ such that $\mathbf{a}$ is a permutation of the $k$-tuple $\left(c_{1}, c_{2}, \ldots, c_{k}\right)$.

Corollary 41. Let $C \subseteq E$ be any subset. Let $m$ be a nonnegative integer. Let $k \in$ $\{1,2, \ldots, m\}$. If $\left(c_{1}, c_{2}, \ldots, c_{m}\right)$ is a greedy $m$-subsequence of $C$, then the number

$$
w\left(c_{k}\right)+\sum_{i=1}^{k-1} d\left(c_{i}, c_{k}\right)
$$

does not depend on the choice of this $m$-subsequence (but only depends on $k$ and on $C$ ).

From now on, the number

$$
w\left(c_{k}\right)+\sum_{i=1}^{k-1} d\left(c_{i}, c_{k}\right)
$$

in Corollary 41 will be denoted by $\nu_{k}(C)$.

Corollary 42. Let $C \subseteq E$ be any subset. Let $m$ be a nonnegative integer. Let $k \in$ $\{1,2, \ldots, m\}$. Let $\left(c_{1}, c_{2}, \ldots, c_{m}\right)$ be a greedy $m$-subsequence of $C$. Let $j \in\{1,2, \ldots, k\}$. Then,

$$
\nu_{k}(C) \leqslant w\left(c_{j}\right)+\sum_{i \in\{1,2, \ldots, k\} \backslash\{j\}} d\left(c_{i}, c_{j}\right) .
$$

Note that Corollary 41 (in the particular case when $w(e)=0$ for all $e \in E$ ) is [7, Conjecture 1], while Theorem 39 (in the same particular case) is [7, Conjecture 2]. 


\subsection{The clone construction}

We shall prove Theorem 39, Theorem 40, Corollary 41 and Corollary 42 by deriving them from the corresponding facts we have already proven about greedy $m$-permutations and maximum-perimeter subsets. This derivation will rely on constructing a larger full ultra triple $(\widehat{E}, \widehat{w}, \widehat{d})$ whose ground set $\widehat{E}$ will contain a sufficiently large number of "clones" of each element of $E$. These "clones" will allow us to transform any $m$-tuple of elements of $E$ into an $m$-tuple of distinct elements of $\widehat{E}$ without disturbing properties like greediness and perimeter.

We construct the new full ultra triple $(\widehat{E}, \widehat{w}, \widehat{d})$ as follows:

- We fix a positive integer $N$. (For now, $N$ can be arbitrary, but later $N$ will be assumed large enough.)

- We let $[N]$ be the set $\{1,2, \ldots, N\}$.

- We define $\widehat{E}$ to be the set $E \times[N]$. It consists of all pairs $(e, i)$ with $e \in E$ and $i \in[N]$.

- We define a function $\widehat{w}: \widehat{E} \rightarrow \mathbb{R}$ by setting

$$
\widehat{w}(e, i)=w(e) \quad \text { for each }(e, i) \in \widehat{E} .
$$

- We define a function $\widehat{d}: \widehat{E} \times \widehat{E} \rightarrow \mathbb{R}$ by setting

$$
\widehat{d}((e, i),(f, j))=d(e, f) \quad \text { for each }(e, i) \in \widehat{E} \text { and }(f, j) \in \widehat{E} .
$$

It is easy to see that $(\widehat{E}, \widehat{w}, \widehat{d})$ is again a full ultra triple. The intuitive meaning of the construction of this full ultra triple is that we have replaced each element $e$ of $E$ by $N$ "clones" $(e, 1),(e, 2), \ldots,(e, N) \in \widehat{E}$; the weights and the mutual distances of these clones are copied over from their originals in $E$. From this point of view, the following lemma should not be surprising:

Lemma 43. Let $C$ be a subset of $E$. Let $\widehat{C}$ be the subset $C \times[N]$ of $\widehat{E}$. Let $m$ be a nonnegative integer. Let $c_{1}, c_{2}, \ldots, c_{m}$ be any $m$ elements of $C$. Let $r_{1}, r_{2}, \ldots, r_{m}$ be any $m$ elements of $[N]$. Then:

(a) We have

$$
\operatorname{PER}\left(\left(c_{1}, r_{1}\right),\left(c_{2}, r_{2}\right), \ldots,\left(c_{m}, r_{m}\right)\right)=\operatorname{PER}\left(c_{1}, c_{2}, \ldots, c_{m}\right)
$$

(Here, the perimeter on the left hand side is computed with respect to the full ultra triple $(\widehat{E}, \widehat{w}, \widehat{d})$, whereas that on the right hand side is computed with respect to the full ultra triple $(E, w, d)$.) 
From now on, assume that $r_{1}, r_{2}, \ldots, r_{m}$ are distinct.

(b) We have

$$
\operatorname{PER}\left\{\left(c_{1}, r_{1}\right),\left(c_{2}, r_{2}\right), \ldots,\left(c_{m}, r_{m}\right)\right\}=\operatorname{PER}\left(c_{1}, c_{2}, \ldots, c_{m}\right)
$$

(c) The $m$-tuple $\left(c_{1}, c_{2}, \ldots, c_{m}\right)$ is a greedy $m$-subsequence of $C$ if and only if the $m$-tuple $\left(\left(c_{1}, r_{1}\right),\left(c_{2}, r_{2}\right), \ldots,\left(c_{m}, r_{m}\right)\right)$ is a greedy m-permutation of $\widehat{C}$.

(d) The $m$-tuple $\left(c_{1}, c_{2}, \ldots, c_{m}\right)$ has maximum perimeter among all $m$-subsequences of $C$ if and only if the set $\left\{\left(c_{1}, r_{1}\right),\left(c_{2}, r_{2}\right), \ldots,\left(c_{m}, r_{m}\right)\right\}$ has maximum perimeter among all $m$-subsets of $\widehat{C}$.

We omit the proof of this lemma, as it is just bookkeeping.

\subsection{Proofs of the analogues}

We are now ready to prove the results promised:

Proof of Theorem 39. Let $k \in\{0,1, \ldots, m\}$.

Pick any positive integer $N$ such that $N \geqslant m$. Define $[N], \widehat{E}, \widehat{w}$ and $\widehat{d}$ as in Subsection 8.4. Pick any $m$ distinct elements $r_{1}, r_{2}, \ldots, r_{m}$ of $[N]$. (These exist because $N \geqslant m$; for example, we can just set $r_{i}=i$.) Let $\widehat{C}$ be the subset $C \times[N]$ of $\widehat{E}$.

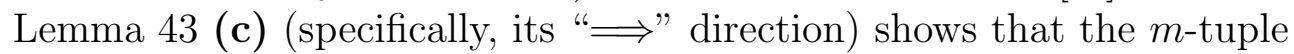
$\left(\left(c_{1}, r_{1}\right),\left(c_{2}, r_{2}\right), \ldots,\left(c_{m}, r_{m}\right)\right)$ is a greedy $m$-permutation of $\widehat{C}$. Thus, Theorem 21 (applied to $(\widehat{E}, \widehat{w}, \widehat{d}), \widehat{C}$ and $\left(c_{i}, r_{i}\right)$ instead of $(E, w, d), C$ and $\left.c_{i}\right)$ shows that the set $\left\{\left(c_{1}, r_{1}\right),\left(c_{2}, r_{2}\right), \ldots,\left(c_{k}, r_{k}\right)\right\}$ has maximum perimeter among all $k$-subsets of $\widehat{C}$. Hence, the "£" direction of Lemma 43 (d) (applied to $k$ instead of $m$ ) shows that the $k$ tuple $\left(c_{1}, c_{2}, \ldots, c_{k}\right)$ has maximum perimeter among all $k$-subsequences of $C$. This proves Theorem 39 .

Our next task is to prove Theorem 40. Before we can do this, let us state a straightforward analogue of Proposition 20 (f) for greedy $k$-subsequences instead of greedy $k$ permutations:

Proposition 44. Let $m$ and $n$ be integers such that $m \geqslant n \geqslant 0$. Let $C$ be a finite nonempty subset of $E$.

If $\left(c_{1}, c_{2}, \ldots, c_{n}\right)$ is a greedy $n$-subsequence of $C$, then we can find $m-n$ elements $c_{n+1}, c_{n+2}, \ldots, c_{m}$ of $C$ such that $\left(c_{1}, c_{2}, \ldots, c_{m}\right)$ is a greedy $m$-subsequence of $C$.

Proof of Proposition 44. Analogous to the proof of Proposition 20 (f). The main difference is that instead of choosing $c_{i} \in C \backslash\left\{c_{1}, c_{2}, \ldots, c_{i-1}\right\}$ that maximizes the perimeter $\operatorname{PER}\left\{c_{1}, c_{2}, \ldots, c_{i}\right\}$ (in the recursive procedure), we now have to choose $c_{i} \in C$ that maximizes the perimeter PER $\left(c_{1}, c_{2}, \ldots, c_{i}\right)$. 
Proof of Theorem 40. Pick any positive integer $N$ such that $N \geqslant m$. Define $[N], \widehat{E}, \widehat{w}$ and $\widehat{d}$ as in Subsection 8.4. Pick any $m$ distinct elements $r_{1}, r_{2}, \ldots, r_{m}$ of $[N]$. (These exist because $N \geqslant m$; for example, we can just set $r_{i}=i$.) Let $\widehat{C}$ be the subset $C \times[N]$ of $\widehat{E}$. Thus, $|\widehat{C}|=|C \times[N]|=\underbrace{|C|}_{\geqslant 1} \cdot \underbrace{|[N]|}_{=N} \geqslant N \geqslant m \geqslant k$.

Recall that $\mathbf{a}$ is a $k$-subsequence of $C$ with maximum perimeter. Write this $k$ subsequence $\mathbf{a}$ in the form $\left(a_{1}, a_{2}, \ldots, a_{k}\right)$. Therefore, the " $\Longrightarrow$ " direction of Lemma 43 (d) (applied to $k$ and $a_{i}$ instead of $m$ and $\left.c_{i}\right)$ shows that the set $\left\{\left(a_{1}, r_{1}\right),\left(a_{2}, r_{2}\right), \ldots,\left(a_{k}, r_{k}\right)\right\}$ has maximum perimeter among all $k$-subsets of $\widehat{C}$. Let us denote this set by $A$. Therefore, Theorem 22 (applied to $(\widehat{E}, \widehat{w}, \widehat{d}), \widehat{C}$ and $k$ instead of $(E, w, d), C$ and $m$ ) shows that there exists a greedy $k$-permutation $\left(\left(c_{1}, q_{1}\right),\left(c_{2}, q_{2}\right), \ldots,\left(c_{k}, q_{k}\right)\right)$ of $\widehat{C}$ such that $A=\left\{\left(c_{1}, q_{1}\right),\left(c_{2}, q_{2}\right), \ldots,\left(c_{k}, q_{k}\right)\right\}$. Consider this greedy $k$-permutation. Hence,

$$
\left\{\left(c_{1}, q_{1}\right),\left(c_{2}, q_{2}\right), \ldots,\left(c_{k}, q_{k}\right)\right\}=A=\left\{\left(a_{1}, r_{1}\right),\left(a_{2}, r_{2}\right), \ldots,\left(a_{k}, r_{k}\right)\right\}
$$

(by the definition of $A$ ). From this, we quickly obtain that the $k$-tuple $\left(a_{1}, a_{2}, \ldots, a_{k}\right)$ must be a permutation of the $k$-tuple $\left(c_{1}, c_{2}, \ldots, c_{k}\right)$ (because $r_{1}, r_{2}, \ldots, r_{k}$ are distinct). In other words, the $k$-tuple $\mathbf{a}$ is a permutation of the $k$-tuple $\left(c_{1}, c_{2}, \ldots, c_{k}\right)$ (since $\mathbf{a}=$ $\left.\left(a_{1}, a_{2}, \ldots, a_{k}\right)\right)$.

Recall again that

$$
\left\{\left(c_{1}, q_{1}\right),\left(c_{2}, q_{2}\right), \ldots,\left(c_{k}, q_{k}\right)\right\}=\left\{\left(a_{1}, r_{1}\right),\left(a_{2}, r_{2}\right), \ldots,\left(a_{k}, r_{k}\right)\right\} .
$$

Hence, $q_{1}, q_{2}, \ldots, q_{k}$ are distinct (since $r_{1}, r_{2}, \ldots, r_{k}$ are distinct). Therefore, the " $\Longleftarrow$ " direction of Lemma 43 (c) (applied to $k$ and $q_{i}$ instead of $m$ and $\left.r_{i}\right)$ shows that $\left(c_{1}, c_{2}, \ldots, c_{k}\right)$ is a greedy $k$-subsequence of $C$ (since $\left(\left(c_{1}, q_{1}\right),\left(c_{2}, q_{2}\right), \ldots,\left(c_{k}, q_{k}\right)\right)$ is a greedy

$k$-permutation of $\widehat{C})$. Since $k \leqslant m$, we can extend this greedy $k$-subsequence to a greedy $m$-subsequence $\left(c_{1}, c_{2}, \ldots, c_{m}\right)$ of $C$ (by Proposition 44 , applied to $n=k$ ). Hence, we have found a greedy $m$-subsequence $\left(c_{1}, c_{2}, \ldots, c_{m}\right)$ of $C$ such that $\mathbf{a}$ is a permutation of the $k$-tuple $\left(c_{1}, c_{2}, \ldots, c_{k}\right)$. This proves Theorem 40 .

Proof of Corollary 41. Pick any positive integer $N$ such that $N \geqslant k$. Define $[N], \widehat{E}, \widehat{w}$ and $\widehat{d}$ as in Subsection 8.4. Let $\widehat{C}$ be the subset $C \times[N]$ of $\widehat{E}$.

Let $\left(c_{1}, c_{2}, \ldots, c_{m}\right)$ be a greedy $m$-subsequence of $C$. Then, $\left(c_{1}, c_{2}, \ldots, c_{k}\right)$ is a greedy $k$-subsequence of $C$ (since the first $k$ entries of any greedy $m$-subsequence form a greedy $k$-subsequence).

Recall the number $\nu_{k}^{\circ}(C)$ we defined just after Corollary 23. Now, consider the number $\nu_{k}^{\circ}(\widehat{C})$ defined in the same fashion, but with respect to the ultra triple $(\widehat{E}, \widehat{w}, \widehat{d})$. We claim that

$$
w\left(c_{k}\right)+\sum_{i=1}^{k-1} d\left(c_{i}, c_{k}\right)=\nu_{k}^{\circ}(\widehat{C}) .
$$


Clearly, proving this will yield Corollary 41.

Pick any $k$ distinct elements $r_{1}, r_{2}, \ldots, r_{k}$ of $[N]$. (These exist because $N \geqslant k$; for example, we can just set $r_{i}=i$.)

Recall that the $k$-tuple $\left(c_{1}, c_{2}, \ldots, c_{k}\right)$ is a greedy $k$-subsequence of $C$. Hence, the $k$ tuple $\left(\left(c_{1}, r_{1}\right),\left(c_{2}, r_{2}\right), \ldots,\left(c_{k}, r_{k}\right)\right)$ is a greedy $k$-permutation of $\widehat{C}$ (by the " $\Longrightarrow$ " direction of Lemma 43 (c), applied to $k$ instead of $m)$. Hence, the definition of $\nu_{k}^{\circ}(\widehat{C})$ yields

$$
\nu_{k}^{\circ}(\widehat{C})=\underbrace{\widehat{w}\left(c_{k}, r_{k}\right)}_{\begin{array}{c}
=w\left(c_{k}\right) \\
\text { (by the definition of } \widehat{w})
\end{array}}+\sum_{i=1}^{k-1} \underbrace{\widehat{d}\left(\left(c_{i}, r_{i}\right),\left(c_{k}, r_{k}\right)\right)}_{\begin{array}{c}
=d\left(c_{i}, c_{k}\right) \\
\text { (by the definition of } \widehat{d})
\end{array}}=w\left(c_{k}\right)+\sum_{i=1}^{k-1} d\left(c_{i}, c_{k}\right) .
$$

This proves (18). Hence, Corollary 41 is proven.

Proof of Corollary 42. This is similar to the above proof of Corollary 41, but now also requires applying Corollary 24 to $(\widehat{E}, \widehat{w}, \widehat{d}), \widehat{C}, k$ and $\left(c_{i}, r_{i}\right)$ instead of $(E, w, d), C, m$ and $c_{i}$. We leave the details to the reader.

Remark 45. Lemma 43 (c) essentially says that, using the full ultra triple $(\widehat{E}, \widehat{w}, \widehat{d})$, we can re-interpret greedy $m$-subsequences as (a certain subclass of) greedy $m$-permutations (as long as $N$ is chosen to satisfy $N \geqslant m$ ).

The reverse direction can also be done: We can re-interpret greedy $m$-permutations of $C$ as greedy $m$-subsequences, as long as $C$ is finite and satisfies $|C| \geqslant m$. To do so, we fix a real number $R$ such that $R>2|\operatorname{PER}(D)|$ for every $D \subseteq C$. We define a new distance function $d_{R}: E \times E \rightarrow \mathbb{R}$ on $E$ by setting

$$
d_{R}(e, f)=\left\{\begin{array}{ll}
d(e, f)+R, & \text { if } e \neq f ; \\
d(e, f), & \text { if } e=f
\end{array} \quad \text { for all } e, f \in E .\right.
$$

It is easy to see that $\left(E, w, d_{R}\right)$ is again a full ultra triple. Moreover, it is easy to see that any $m$-subsequence of $C$ containing two equal entries has smaller perimeter with respect to $\left(E, w, d_{R}\right)$ than any $m$-subset of $C$. Hence, the maximum perimeter of an $m$ subsequence of $C$ with respect to $\left(E, w, d_{R}\right)$ can only be achieved by an $m$-subsequence with no equal entries. Hence, this maximum perimeter is the maximum perimeter of an $m$-subset of $C$ with respect to $\left(E, w, d_{R}\right)$. Meanwhile, the perimeter of an $m$-subset of $C$ with respect to $\left(E, w, d_{R}\right)$ equals its perimeter with respect to the original full ultra triple $(E, w, d)$ plus the constant $\left(\begin{array}{c}m \\ 2\end{array}\right) R$. Hence, the $m$-subsets of $C$ having maximum perimeter with respect to $\left(E, w, d_{R}\right)$ are precisely the same that have maximum perimeter with respect to $(E, w, d)$. From this, it is easy to see that the greedy $m$-subsequences of $C$ with respect to $\left(E, w, d_{R}\right)$ are precisely the greedy $m$-permutations of $C$ with respect to $(E, w, d)$.

When $|C|<m$, this reasoning no longer works, since every $m$-subsequence of $C$ has two equal entries (and there are no $m$-subsets of $C$ ). In this case, the greedy $m$-subsequences 
of $C$ with respect to $\left(E, w, d_{R}\right)$ can be informally regarded as greedy $m$-subsequences of $C$ with respect to $(E, w, d)$ that defer picking identical entries as long as they can (in a sense).

\section{Relation to Bhargava's $\boldsymbol{P}$-orderings}

Let us now connect greedy $m$-permutations with the concept of $P$-orderings introduced by Manjul Bhargava in [2, Section 2]. (The notions of $p$-orderings in [3, Section 4] and [4, Section 2] are particular cases.) This connection was already noticed by Bhargava (see the paragraph after the proof of Lemma 2 in [2]), who, however, never elaborated on it or made any further inroads into the study of general ultra triples.

We fix a Dedekind $\operatorname{ring}^{18} R$ and a nonzero prime ideal $P$ of $R$. For any nonzero $a \in R$, we let $v_{P}(a)$ denote the highest ${ }^{19} k \in \mathbb{N}$ that satisfies $a \in P^{k}$. (Equivalently, $v_{P}(a)$ is the exponent with which $P$ appears in the factorization of the principal ideal $a R$ into prime ideals. ${ }^{20}$ ) We also set $v_{P}(0)=+\infty$. Thus, an element $v_{P}(a) \in \mathbb{N} \cup\{+\infty\}$ is defined for every $a \in R$. Moreover, the map $v_{P}: R \rightarrow \mathbb{N} \cup\{+\infty\}$ satisfies

$$
v_{P}(a b)=v_{P}(a)+v_{P}(b) \quad \text { and } \quad v_{P}(a+b) \leqslant \max \left\{v_{P}(a), v_{P}(b)\right\}
$$

for all $a, b \in R$.

The simplest example for this is when $R=\mathbb{Z}$ and $P=p \mathbb{Z}$ for some prime number $p$. In this case, $v_{P}(a)=v_{p}(a)$, where $v_{p}(a)$ is defined as in Example 4. This particular case is the one studied in [3, Section 4] and [4, Section 2].

Furthermore, we fix a nonempty subset $E$ of $R$. (Bhargava denotes this subset by $X$ instead.) Now, Bhargava defines a P-ordering of $E$ to be a sequence $\left(a_{0}, a_{1}, a_{2}, \ldots\right)$ of elements of $E$ defined recursively as follows: For each $k \in \mathbb{N}$, we define $a_{k}$ (assuming that $a_{0}, a_{1}, \ldots, a_{k-1}$ are already defined) to be an element of $E$ minimizing the quantity

$$
v_{P}\left(\left(a_{k}-a_{0}\right)\left(a_{k}-a_{1}\right) \cdots\left(a_{k}-a_{k-1}\right)\right) .
$$

Note that the quantity (19) indeed attains its minimum at some (usually non-unique) $a_{k} \in E$, since it is an element of the well-ordered set $\mathbb{N} \cup\{+\infty\}$.

We now claim that this notion of $P$-ordering is almost a particular case of the notion of a greedy $m$-permutation for a certain ultra triple. Some amount of work is necessary to bridge the technical discrepancies between these two notions:

\footnotetext{
${ }^{18}$ See [12, Chapter 1] or [1, Chapter 3] for an introduction to Dedekind rings. In a nutshell, a Dedekind ring is an integral domain in which every nonzero ideal has a unique factorization into a product of prime ideals. Other equivalent definitions of Dedekind rings exist. Dedekind rings are also known as Dedekind domains.

For our purposes, it suffices to know that $\mathbb{Z}$ is a Dedekind ring; the examples it provides are sufficiently rich in substance that greater generality is not strictly necessary.

${ }^{19}$ Here and in the following, we set $\mathbb{N}=\{0,1,2, \ldots\}$.

${ }^{20}$ The equivalence between these two definitions of $v_{P}(a)$ follows from [1, Corollary 3.3.3]; this also proves that the first definition is valid (i.e., there exists a highest $k \in \mathbb{N}$ that satisfies $a \in P^{k}$ ).
} 
First of all, $P$-orderings are infinite sequences, whereas greedy $m$-permutations are $m$-tuples. To bring them closer together, we fix an $m \in \mathbb{N}$, and we define an $(P, m)$ ordering of $E$ to be an $m$-tuple $\left(a_{0}, a_{1}, \ldots, a_{m-1}\right)$ of elements of $E$ such that for each $k \in$ $\{0,1, \ldots, m-1\}$, the element $a_{k}$ of $E$ minimizes the quantity (19) (where $a_{0}, a_{1}, \ldots, a_{k-1}$ are considered fixed). Clearly, the first $m$ entries of any $P$-ordering form a $(P, m)$-ordering, and conversely, any $(P, m)$-ordering can be extended to a $P$-ordering. Thus, if we want to study (finitary) properties of $P$-orderings, it suffices to understand $(P, m)$-orderings. Thus we are back in the realm of finite sequences.

We furthermore notice something simple:

Lemma 46. Let $C$ be a subset of $E$, and let $m$ be a nonnegative integer such that $|C| \geqslant m$. Then, any $(P, m)$-ordering of $C$ is an $m$-tuple of distinct elements.

Proof of Lemma 46. Any $(P, m)$-ordering $\left(a_{0}, a_{1}, \ldots, a_{m-1}\right)$ of $C$ can be constructed recursively as follows: For each $k \in\{0,1, \ldots, m-1\}$, we define $a_{k}$ (assuming that the elements $a_{0}, a_{1}, \ldots, a_{k-1}$ are already defined) to be an element of $C$ minimizing the quantity (19). But this quantity (19) is $+\infty$ when $a_{k}$ equals one of $a_{0}, a_{1}, \ldots, a_{k-1}$, and otherwise is a nonnegative integer. In order to minimize this quantity, we must thus pick $a_{k}$ distinct from $a_{0}, a_{1}, \ldots, a_{k-1}$ (as long as such an $a_{k}$ exists; but it does exist, since $k \leqslant m-1<m \leqslant|C|)$. Hence, $a_{0}, a_{1}, \ldots, a_{m-1}$ are distinct. This proves Lemma 46.

Next, we define an ultra triple $\left(E, w, d^{\prime}\right)$ as follows: We define the weight function $w: E \rightarrow \mathbb{R}$ by setting $w(e)=0$ for all $e \in E$. We define a map $d^{\prime}: E \times E \rightarrow \mathbb{R}$ by setting

$$
d^{\prime}(a, b)=-v_{P}(a-b) \quad \text { for all }(a, b) \in E \underline{\times} E .
$$

(This generalizes the map $d^{\prime}$ from Example 5.)

Now, $\left(E, w, d^{\prime}\right)$ is an ultra triple. Throughout this section, we shall always be using this ultra triple (when we speak, e.g., of greedy $m$-permutations). We claim the following:

Proposition 47. Let $C$ be a subset of $E$. Let $m \in \mathbb{N}$. Let $c_{1}, c_{2}, \ldots, c_{m} \in C$ be distinct. Then, the $m$-tuple $\left(c_{1}, c_{2}, \ldots, c_{m}\right)$ is a greedy m-permutation of $C$ if and only if it is a $(P, m)$-ordering of $C$.

Proof of Proposition 4\%. We have $|C| \geqslant m$ (since $C$ has at least the $m$ distinct elements $\left.c_{1}, c_{2}, \ldots, c_{m}\right)$.

The entries $c_{1}, c_{2}, \ldots, c_{m}$ of the $m$-tuple $\left(c_{1}, c_{2}, \ldots, c_{m}\right)$ are distinct. Hence, the definition of a greedy $m$-permutation yields the following:

Claim 1: The $m$-tuple $\left(c_{1}, c_{2}, \ldots, c_{m}\right)$ is a greedy $m$-permutation of $C$ if and only if for each $i \in\{1,2, \ldots, m\}$ and each $x \in C \backslash\left\{c_{1}, c_{2}, \ldots, c_{i-1}\right\}$, the inequality (2) holds.

On the other hand, the definition of a $(P, m)$-ordering shows that $\left(c_{1}, c_{2}, \ldots, c_{m}\right)$ is a $(P, m)$-ordering of $C$ if and only if for each $k \in\{0,1, \ldots, m-1\}$, the element $c_{k+1}$ of $C$ minimizes the quantity $v_{P}\left(\left(c_{k+1}-c_{1}\right)\left(c_{k+1}-c_{2}\right) \cdots\left(c_{k+1}-c_{k}\right)\right)$ (where $c_{1}, c_{2}, \ldots, c_{k}$ are 
considered fixed). Substituting $i-1$ for $k$ in this statement, we obtain the following: The $m$-tuple $\left(c_{1}, c_{2}, \ldots, c_{m}\right)$ is a $(P, m)$-ordering of $C$ if and only if for each $i \in\{1,2, \ldots, m\}$, the element $c_{i}$ of $C$ minimizes the quantity $v_{P}\left(\left(c_{i}-c_{1}\right)\left(c_{i}-c_{2}\right) \cdots\left(c_{i}-c_{i-1}\right)\right)$ (where $c_{1}, c_{2}, \ldots, c_{i-1}$ are considered fixed). We can restate this as follows:

Claim 2: The $m$-tuple $\left(c_{1}, c_{2}, \ldots, c_{m}\right)$ is a $(P, m)$-ordering of $C$ if and only if for each $i \in\{1,2, \ldots, m\}$ and each $x \in C$, the inequality

$$
\begin{aligned}
& v_{P}\left(\left(c_{i}-c_{1}\right)\left(c_{i}-c_{2}\right) \cdots\left(c_{i}-c_{i-1}\right)\right) \\
& \leqslant v_{P}\left(\left(x-c_{1}\right)\left(x-c_{2}\right) \cdots\left(x-c_{i-1}\right)\right)
\end{aligned}
$$

holds.

Note that if $i \in\{1,2, \ldots, m\}$ and $x \in\left\{c_{1}, c_{2}, \ldots, c_{i-1}\right\}$, then the inequality (20) automatically holds ${ }^{21}$. Therefore, if $i \in\{1,2, \ldots, m\}$ is given, then the inequality (20) holds for each $x \in C$ if and only if it holds for each $x \in C \backslash\left\{c_{1}, c_{2}, \ldots, c_{i-1}\right\}$. Hence, in Claim 2, we can replace "each $x \in C$ " by "each $x \in C \backslash\left\{c_{1}, c_{2}, \ldots, c_{i-1}\right\}$ ". Thus, Claim 2 rewrites as follows:

Claim 3: The $m$-tuple $\left(c_{1}, c_{2}, \ldots, c_{m}\right)$ is a $(P, m)$-ordering of $C$ if and only if for each $i \in\{1,2, \ldots, m\}$ and each $x \in C \backslash\left\{c_{1}, c_{2}, \ldots, c_{i-1}\right\}$, the inequality (20) holds.

For any $i \in\{1,2, \ldots, m\}$ and $x \in C \backslash\left\{c_{1}, c_{2}, \ldots, c_{i-1}\right\}$, we have the following chain of logical equivalences:

(the inequality (2) holds)

$$
\begin{aligned}
& \Longleftrightarrow\left(\operatorname{PER}\left\{c_{1}, c_{2}, \ldots, c_{i}\right\} \geqslant \operatorname{PER}\left\{c_{1}, c_{2}, \ldots, c_{i-1}, x\right\}\right) \\
& \Longleftrightarrow\left(w\left(c_{i}\right)+\sum_{j=1}^{i-1} d^{\prime}\left(c_{i}, c_{j}\right) \geqslant w(x)+\sum_{j=1}^{i-1} d^{\prime}\left(x, c_{j}\right)\right) \\
& \quad\left(\begin{array}{c}
\text { here, we have subtracted PER } \left.\left\{c_{1}, c_{2}, \ldots, c_{i-1}\right\}\right) \\
\text { from both sides of the inequality }
\end{array}\right) \\
& \Longleftrightarrow\left(\sum_{j=1}^{i-1} d^{\prime}\left(c_{i}, c_{j}\right) \geqslant \sum_{j=1}^{i-1} d^{\prime}\left(x, c_{j}\right)\right) \quad(\text { since } w(e)=0 \text { for all } e \in E) \\
& \Longleftrightarrow\left(\sum_{j=1}^{i-1} v_{P}\left(c_{i}-c_{j}\right) \leqslant \sum_{j=1}^{i-1} v_{P}\left(x-c_{j}\right)\right) \quad\left(\text { by the definition of } d^{\prime}\right) \\
& \Longleftrightarrow\left(v_{P}\left(\prod_{j=1}^{i-1}\left(c_{i}-c_{j}\right)\right) \leqslant v_{P}\left(\prod_{j=1}^{i-1}\left(x-c_{j}\right)\right)\right)
\end{aligned}
$$

\footnotetext{
${ }^{21}$ because in this case, we have $\left(x-c_{1}\right)\left(x-c_{2}\right) \cdots\left(x-c_{i-1}\right)=0$ and thus $v_{P}\left(\left(x-c_{1}\right)\left(x-c_{2}\right) \cdots\left(x-c_{i-1}\right)\right)=v_{P}(0)=+\infty$
} 


$$
\begin{aligned}
& \left(\begin{array}{c}
\text { since } \sum_{j \in J} v_{P}\left(a_{j}\right)=v_{P}\left(\prod_{j \in J} a_{j}\right) \\
\text { for any finite family }\left(a_{j}\right)_{j \in J} \text { of elements of } R
\end{array}\right) \\
& \Longleftrightarrow \text { (the inequality (20) holds) }
\end{aligned}
$$

(since $\prod_{j=1}^{i-1}\left(c_{i}-c_{j}\right)=\left(c_{i}-c_{1}\right)\left(c_{i}-c_{2}\right) \cdots\left(c_{i}-c_{i-1}\right)$ and $\left.\prod_{j=1}^{i-1}\left(x-c_{j}\right)=\left(x-c_{1}\right)\left(x-c_{2}\right) \cdots\left(x-c_{i-1}\right)\right)$.

Now, we have the following chain of logical equivalences:

$$
\begin{aligned}
& \left(\left(c_{1}, c_{2}, \ldots, c_{m}\right) \text { is a greedy } m \text {-permutation of } C\right) \\
& \Longleftrightarrow((2) \text { holds for each } i \in\{1,2, \ldots, m\} \\
& \text { and each } \left.x \in C \backslash\left\{c_{1}, c_{2}, \ldots, c_{i-1}\right\}\right) \quad \text { (by Claim 1) } \\
& \Longleftrightarrow((20) \text { holds for each } i \in\{1,2, \ldots, m\} \\
& \text { and each } \left.\left.x \in C \backslash\left\{c_{1}, c_{2}, \ldots, c_{i-1}\right\}\right) \quad \text { (by }(21)\right) \\
& \left.\Longleftrightarrow\left(\left(c_{1}, c_{2}, \ldots, c_{m}\right) \text { is a }(P, m) \text {-ordering of } C\right) \quad \text { (by Claim } 3\right) \text {. }
\end{aligned}
$$

Hence, the $m$-tuple $\left(c_{1}, c_{2}, \ldots, c_{m}\right)$ is a greedy $m$-permutation of $C$ if and only if it is a $(P, m)$-ordering of $C$. This proves Proposition 47 .

Equipped with Proposition 47, we can now translate each result about greedy $m$ permutations into the language of $(P, m)$-orderings as long as $|C| \geqslant m$ (because Lemma 46 shows that any $(P, m)$-ordering consists of distinct entries in this case) ${ }^{22}$ In particular, Corollary 23 becomes [2, Theorem 1], while Corollary 24 becomes [2, Lemma 2]. (More precisely, we obtain the analogues of $[2$, Theorem 1] and [2, Lemma 2] for $(P, m)$-orderings instead of $P$-orderings. But since the first $m$ entries of any $P$-ordering form a $(P, m)$ ordering, these analogues immediately yield [2, Theorem 1] and [2, Lemma 2].)

We note in passing that the " $P$-orderings of order $h$ " defined in $[4$, Section 2.2] can also be regarded as a particular case of greedy $m$-permutations (up to the already mentioned technicalities); we only need to modify the distance function $d^{\prime}$.

\section{Appendix: Greediness of $(1,2, \ldots, m)$ for $p$-adic metrics}

In this section, we shall prove the claim made in Example 18.

Actually we prove the following more general result

Proposition 48. Let $\mathbb{N}, c, \mathbf{r}=\left(r_{0}, r_{1}, r_{2}, \ldots\right), v_{\mathbf{r}}(x), E$ and $d$ be as in Example 6 . Assume that $d(a, b)$ is well-defined for each $(a, b) \in E \underline{\times} E$. Assume furthermore that $E$ contains $1,2, \ldots, m$. We define $w: E \rightarrow \mathbb{R}$ by setting $w(e)=0$ for each $e \in E$.

Then,$(1,2, \ldots, m)$ is a greedy $m$-permutation of $E$.

\footnotetext{
${ }^{22}$ The case $|C|<m$ is a degenerate case which can easily be reduced to the case $|C| \geqslant m$ by focussing only on the first $|C|$ many entries of the $(P, m)$-ordering. (All the other entries merely repeat the first $|C|$ many entries, in an arbitrary way, so there is nothing of interest to say about them.)
} 
Proof. Let $\mathbf{1}(\mathcal{A})$ denote the truth value of any statement $\mathcal{A}$. The weakly decreasing function $c: \mathbb{N} \rightarrow \mathbb{R}$ may be represented as

$$
c(x)=\alpha_{0}+\sum_{i=1}^{\infty} \alpha_{i} \mathbf{1}(x \geqslant i)
$$

with $\alpha_{0}=c(0)$ and nonpositive coefficients $\alpha_{i}=c(i)-c(i-1)$ for $i \geqslant 1$. (The sum in $(22)$ is eventually finite, thus well-defined, for every $x \in \mathbb{N}$.) The Proposition claims that for each $k=1, \ldots, m$, the sum

$$
\operatorname{PER}\{1,2, \ldots, k-1, x\}-\operatorname{PER}\{1,2, \ldots, k-1\}=\sum_{j=1}^{k-1} c\left(v_{\mathbf{r}}(x-j)\right)
$$

(where $x$ varies on $\{k, k+1, \ldots\}$ ) is maximized for $x=k$. Due to representation (22), it suffices to prove that for each $i \geqslant 1$ the sum

$$
A(x):=\sum_{j=1}^{k-1} \mathbf{1}\left(v_{\mathbf{r}}(x-j) \geqslant i\right)=\sum_{j=1}^{k-1} \mathbf{1}\left(r_{i} \text { divides } x-j\right)
$$

is minimized for $x=k$. This is clear: $A(k)=\left\lfloor(k-1) / r_{i}\right\rfloor$, and $A(x) \geqslant\left\lfloor(k-1) / r_{i}\right\rfloor$ for every integer $x$, since the set $\{x-1, x-2, \ldots, x-(k-1)\}$ contains $\left\lfloor(k-1) / r_{i}\right\rfloor$ disjoint intervals of $r_{i}$ consecutive integers each, and each such interval contains a number divisible by $r_{i}$.

\section{Acknowledgements}

The authors thank a referee for some helpful comments. DG also thanks the Mathematisches Forschungsinstitut Oberwolfach for its hospitality in 2019, during which some early parts of this paper have been written.

\section{References}

[1] Robert B. Ash, A Course In Algebraic Number Theory. https://faculty.math. illinois.edu/ r-ash/ANT.html

[2] Manjul Bhargava, P-orderings and polynomial functions on arbitrary subsets of Dedekind rings, J. reine. angew. Math. 490 (1997), 101-127. http://resolver. sub.uni-goettingen.de/purl?PPN243919689_0490

[3] Manjul Bhargava, The Factorial Function and Generalizations, The American Mathematical Monthly, Vol. 107, No. 9 (Nov., 2000), pp. 783-799. https://doi.org/10. $2307 / 2695734$

[4] Manjul Bhargava, On P-orderings, rings of integer-valued polynomials, and ultrametric analysis, Journal of the American Mathematical Society Vol. 22, No. 4 (October 2009), pp. 963-993. https://www.jstor.org/stable/40587258 
[5] Peter A. Brooksbank, Transversal Greedoids, Europ. J. Combinatorics (1997) 18, pp. 137-141. https://doi.org/10.1006/eujc.1992.0072

[6] Victor Bryant, Ian Sharpe, Gaussian, Strong and Transversal Greedoids, European Journal of Combinatorics 20, Issue 4, May 1999, pp. 259-262. https://doi.org/ 10.1006/eujc.1998.0287

[7] Darij Grinberg, MathOverflow question \#314130. https://mathoverflow.net/ questions/314130/

[8] Darij Grinberg, The Bhargava greedoid as a Gaussian elimination greedoid, arXiv:2001.05535v2. https://arxiv.org/abs/2001.05535v2

[9] Markus Kloock, Das Twisten von Matroiden, $\mathrm{PhD}$ thesis at the Universität zu Köln, 2003. https://kups.ub.uni-koeln.de/1031/

[10] Bernhard Korte, László Lovász, Rainer Schrader, Greedoids, Algorithms and Combinatorics \#4, Springer 1991.

[11] Vincent Moulton, Charles Semple, Mike Steel, Optimizing phylogenetic diversity under constraints, Journal of Theoretical Biology 246 (2007), pp. 186-194. https: //doi.org/10.1016/j.jtbi.2006.12.021

[12] Wladyslaw Narkiewicz, Elementary and Analytic Theory of Algebraic Numbers, 3rd edition, Springer 2004. https://doi .org/10.1007/978-3-662-07001-7

[13] James Oxley, Matroid theory, Oxford Graduate Texts in Mathematics \#21, 2nd edition, Oxford University Press 2011. 